\title{
WHEN IS ZERO IN THE NUMERICAL RANGE OF A COMPOSITION OPERATOR?
}

\author{
PAUL S. BOURDON AND JOEL H. SHAPIRO
}

\begin{abstract}
We work on the Hardy space $H^{2}$ of the open unit disc $\mathbb{U}$, and consider the numerical ranges of composition operators $C_{\varphi}$ induced by holomorphic self-maps $\varphi$ of $\mathbb{U}$. For maps $\varphi$ that fix a point of $\mathbb{U}$ we determine precisely when 0 belongs to the numerical range $W$ of $C_{\varphi}$, and in the process discover the following dichotomy: either $0 \in W$ or the real part of $C_{\varphi}$ admits a decomposition that reveals it to be strictly positive-definite. In this latter case we characterize those operators that are sectorial. For compact composition operators our work has the following consequences: it yields a complete description of the corner points of the closure of $W$, and it establishes when $W$ is closed. In the course of our investigation we uncover surprising connections between composition operators, Chebyshev polynomials, and Pascal matrices.
\end{abstract}

\section{INTRODUCTION}

The study of composition operators turns the lens of operator theory upon the classical theory of holomorphic self-maps of the open unit disc. Let $\mathbb{U}$ denote this disc, and $H(\mathbb{U})$ the space of all functions holomorphic thereon. The idea is to associate with each holomorphic $\varphi: \mathbb{U} \rightarrow \mathbb{U}$ the composition operator $C_{\varphi}$ defined on $H(\mathbb{U})$ by the equation

$$
C_{\varphi} f=f \circ \varphi \quad(f \in H(\mathbb{U})) .
$$

The operator so defined is linear on $H(\mathbb{U})$, and continuous in the natural (compact-open) topology of that space; the goal is to connect the function-theoretic properties of $\varphi$ with the operator-theoretic behavior of $C_{\varphi}$.

The subject's true appeal originates with J.E. Littlewood's remarkable Subordination Theorem [24, 1925], which guarantees that each composition operator restricts to a bounded linear transformation on the Hardy space $H^{2}$ of $\mathbb{U}$. Littlewood's Theorem provides a striking way of connecting the function theory of the unit disc with the theory of bounded linear operators on Hilbert space. The past three decades have witnessed enthusiastic exploration of this connection, as partially recorded in the monographs [8] and [32], and the recent conference proceedings [17].

In this paper we study how the numerical range of a composition operator on $H^{2}$ is influenced by the properties of its inducing map. The numerical range of a bounded linear operator $T$ on a Hilbert space $\mathcal{H}$ is the set of complex numbers

$$
W(T)=\{\langle T f, f\rangle: f \in \mathcal{H},\|f\|=1\} .
$$

Some important properties of the numerical range are:

Date: October 17, 2000.

This research was supported in part by the National Science Foundation. 
(a) it lies in the disc $\{|w| \leq\|T\|\}$ (obvious);

(b) it contains every eigenvalue of $T$ (also obvious);

(c) its closure contains the spectrum of $T$ (elementary, see e.g., [15, Theorem 1.2-1, p. 6] or [18, Problem 214, p. 115])

(d) it is not similarity invariant (elementary, but a major theme in this paper);

(e) it is convex (very mysterious!).

Note in particular that according to property (b) the numerical range of any composition operator contains the point 1 , since 1 is an eigenvalue (composition operators fix constant functions). Also note that by properties (c) and (e), the convex hull of the spectrum of $T$ is contained in the closure of $W(T)$ for any Hilbert space operator $T$.

Convexity was first proved for the boundary of the numerical range by Toeplitz in 1918 [34], and the result was completed the following year by Hausdorff [19]. This ToeplitzHausdorff Theorem has fascinated mathematicians ever since. Many proofs have been given, perhaps the simplest being that of Dekker [12] (see [18, Problem 210]), and the most conceptual that of Davis [9]. However much remains to be understood; for example, the numerical range is easily seen to be compact if $\mathcal{H}$ is finite dimensional, but for $\mathcal{H}$ merely separable the numerical range was only relatively recently shown (by Agler) to be a Borel set $[1,1982]$.

In this paper we focus on the following:

Zero-Inclusion Question. For which $\varphi$ does $W\left(C_{\varphi}\right)$ contain the origin?

We are drawn to this question for three reasons:

- The numerical range of any composition operator, except for the identity, contains the origin in its closure (Theorem 3.1 below).

- The numerical range of a compact operator is closed if and only if it contains the origin [11].

- Resolution of the Zero-Inclusion Question for $W\left(C_{\varphi}\right)$ leads to a description of the corner points of the closure of $W\left(C_{\varphi}\right)$ when $C_{\varphi}$ is compact (Theorem 7.5 below).

When $C_{\varphi}$ is compact, $\varphi$ necessarily fixes a point in $\mathbb{U}$ (see, e.g., [32, p. 84]). For those symbols $\varphi$ that fix a point $p \in \mathbb{U}$ we answer the Zero-Inclusion Question completely (Theorem 5.3): 0 does not belong to $W\left(C_{\varphi}\right)$ if and only if there is an $r \in(0,1]$ such that

$$
|p| \leq \sqrt{r} \text { and } \varphi(z)=\alpha_{p}\left(r \alpha_{p}(z)\right) \quad(z \in \mathbb{U}),
$$

where $\alpha_{p}$ is the self-inverse automorphism of $\mathbb{U}$ given by $\alpha_{p}(z)=(p-z) /(1-\bar{p} z)$. In addition, we show that $0 \in W\left(C_{\varphi}\right)$ implies $0 \in \operatorname{int} W\left(C_{\varphi}\right)$ as long as $\varphi$ doesn't have the form $\varphi(z)=t z$ for some $t \in[-1,0]$.

Our work on the Zero-Inclusion Question occupies Sections 3, 4, and 5 of this paper. This work is completed in Section 5, where we consider composition operators whose symbols have the form

$$
\varphi=\alpha_{p} \circ\left(r \alpha_{p}\right) \text {, for some } p \in \mathbb{U} \text { and } r \in(0,1) .
$$

We emphasize that for fixed $r$ each of these "conformal dilations" $\varphi$ is conformally conjugate to the ordinary dilation $z \rightarrow r z$; hence, the corresponding composition operators $C_{\varphi}$ are similar to the simple composition operator $f(z) \rightarrow f(r z)$ whose numerical range does not include zero (it is easily seen to be the interval $(0,1])$. Despite the similarity of these 
operators, there is, as we indicated in the preceding paragraph, a zero-inclusion dichotomy: $0 \notin W\left(C_{\varphi}\right)$ if $|p| \leq \sqrt{r}$ and $0 \in \operatorname{int} W\left(C_{\varphi}\right)$ if $|p|>\sqrt{r}$. This points out the crucial role played in our work by the fact that the numerical range is not similarity-invariant. Both this lack of invariance and the zero-inclusion dichotomy are illustrated in the following sequence of figures, the second and third of which are computer-generated approximations. In all three figures $\varphi=\alpha_{p} \circ\left(r \alpha_{p}\right)$ with $r=1 / 4$ : in the first figure $p=0$, i.e. $\varphi$ is a dilation; in the second one $p=3 / 8$, so that $p<\sqrt{r}$; and in the third $p=3 / 4$, illustrating the behavior for $p>\sqrt{r}$.

NUMERICAL RANGES OF THREE SIMILAR COMPOSITION OPERATORS

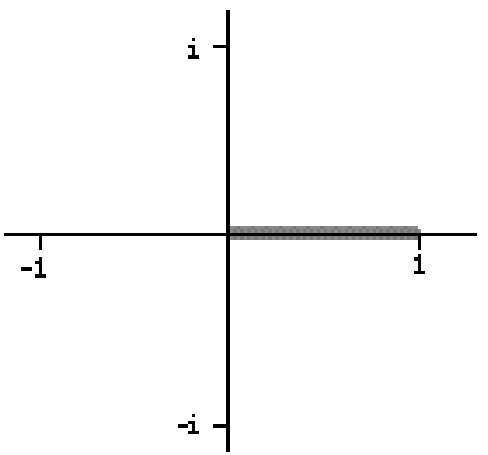

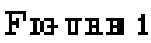

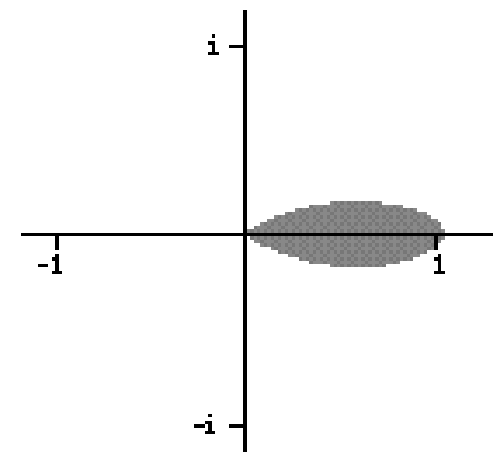

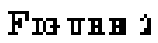

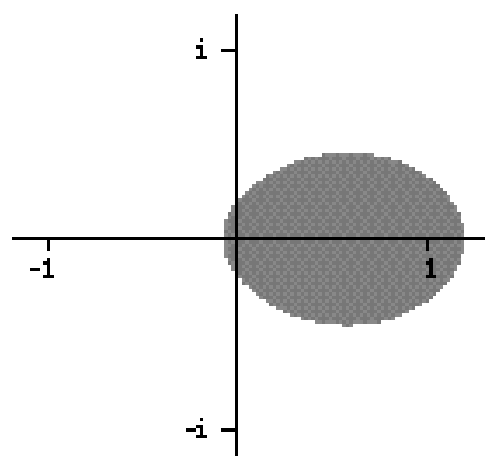

FI?

Observe that in Figure 2, $W\left(C_{\varphi}\right)$ appears to lie in the open right half-plane. Our work on the zero-inclusion dichotomy in Section 5 shows that Figure 2 representative: we prove that that the real part of $C_{\varphi}$ is strictly positive whenever $\varphi$ has the form (1) and $|p| \leq \sqrt{r}$.

In Section 6 we continue our analysis of $W\left(C_{\varphi}\right)$ for $\varphi$ conformally conjugate to a positive dilation. We prove that when $\varphi$ has the form $(1)$ and $|p|<r$, then not only does $W\left(C_{\varphi}\right)$ lie in the open right half-plane, it lies in a sector $\{z:|\arg (z)|<\theta\}$ for some positive acute angle $\theta$ (Figure 2 is, once again, representative). However, we show that when $|p|=\sqrt{r}$, then $W\left(C_{\varphi}\right)$ does not lie in such a sector. We thus obtain a characterization of the sectorial composition operators (among those whose symbols fix a point in $\mathbb{U}$ ). Behind our analysis of the zero-inclusion and sectorial issues at the point $p=\sqrt{r}$ lie interesting connections between composition operators, Pascal's matrix, and Chebyshev polynomials.

In Section 7 we show how our work on the Zero-Inclusion Question leads to a complete characterization of the corner points of the closure of $W\left(C_{\varphi}\right)$ when $C_{\varphi}$ is compact. (An element $p$ of a set $E \subset \mathbb{C}$ is a corner point of $E$ provided $E$ is contained in a half-cone with vertex $p$ and angular opening less than $\pi$.)

In the final section of this paper we discuss the Zero-Inclusion Question for mappings $\varphi$ that do not fix a point of $\mathbb{U}$. Our work in this section and in several others is simplified by the following result (Corollary 3.6), which requires no fixed-point hypothesis:

Suppose $\varphi$ is a nonconstant holomorphic self-map of $\mathbb{U}$. If $\varphi$ is not one-to-one, then $0 \in \operatorname{int} W\left(C_{\varphi}\right)$. 
We remark that Matache, who studies numerical ranges of composition operators in [26], presents some results (Theorems 3.1, 3.4, and 3.5) whose conclusions imply $0 \in \operatorname{int} W\left(C_{\varphi}\right)$; in each of these results $\varphi$ is not univalent.

Acknowledgment. We wish to thank Wayne Dymacek of Washington and Lee University for providing us with helpful references concerning Pascal matrices and Chebyshev polynomials.

\section{Preliminaries}

We gather here, often without proof, some well known results about Hardy spaces, numerical ranges, and conformal automorphisms that will be needed for the sequel.

2.1. The space $H^{2}$. For functions $f$ holomorphic on $\mathbb{U}$ we use $\hat{f}(n)$ to denote $n$-th coefficient of $f$ in its Taylor expansion about the origin. The Hardy space $H^{2}$ is the collection of functions $f$ holomorphic on $\mathbb{U}$ with

$$
\|f\|^{2}:=\sum_{n=0}^{\infty}|\hat{f}(n)|^{2}<\infty .
$$

The function $\|\cdot\|$ so defined is a norm that turns $H^{2}$ into a Hilbert space with inner product

$$
\langle f, g\rangle=\sum_{n=0}^{\infty} \hat{f}(n) \overline{\hat{g}(n)} .
$$

By the Riesz-Fisher theorem the trigonometric series $\sum_{0}^{\infty} \hat{f}(n) e^{i n \theta}$ associated with an $H^{2}$ function $f$ is the Fourier series of some function $f^{*} \in L^{2}=L^{2}(m, \partial \mathbb{U})$, where $m$ denotes arclength measure on $\partial \mathbb{U}$, normalized to have total mass one. Clearly the map $f \rightarrow f^{*}$ takes $H^{2}$ isometrically onto the closed subspace of $L^{2}$ consisting of functions whose Fourier coefficients of negative index vanish. The boundary function $f^{*}$ is, in fact, the natural extension of $f$ to $\partial \mathbb{U}$; it is just the radial limit function

$$
f^{*}(\zeta)=\lim _{r \rightarrow 1-} f(r \zeta)
$$

where the limit exists for $m$ almost every $\zeta \in \partial \mathbb{U}$. From now on we simply write $f(\zeta)$ instead of $f^{*}(\zeta)$ for each $\zeta \in \partial \mathbb{U}$ at which this radial limit exists, letting the context determine the meaning of the symbol $f$.

With these observations the norm and inner product in $H^{2}$ can be computed on the boundary of the unit disc as:

$$
\|f\|^{2}=\int_{\partial \mathbb{U}}|f|^{2} d m \quad\left(f \in H^{2}\right)
$$

and

$$
\langle f, g\rangle=\int_{\partial \mathbb{U}} f \bar{g} d m \quad\left(f, g \in H^{2}\right)
$$


2.2. Reproducing kernels. Associated with each point $a \in \mathbb{U}$ is its reproducing kernel

$$
K_{a}(z):=\frac{1}{1-\bar{a} z}=\sum_{n=0}^{\infty} \bar{a}^{n} z^{n} \quad(z \in \mathbb{U})
$$

Clearly $K_{a} \in H^{2}$ for every $a \in \mathbb{U}$, and the series definition of the inner product for $H^{2}$ yields immediately the reproducing property

$$
\left\langle f, K_{a}\right\rangle=f(a) \quad\left(f \in H^{2}, a \in \mathbb{U}\right) .
$$

The importance for composition operators of the collection of reproducing kernels stems in part from the adjoint property

$$
C_{\varphi}^{*} K_{a}=K_{\varphi(a)} \quad(a \in \mathbb{U}),
$$

which follows quickly from the reproducing property.

2.3. Compression and the numerical range. We have already defined the numerical range $W(T)$ of an operator $T$ on a Hilbert space $\mathcal{H}$ and noted its salient features. $W(T)$ is just the image of the unit sphere of $\mathcal{H}$ under the quadratic form $Q_{T}: \mathcal{H} \rightarrow \mathbb{C}$ associated with $T$ :

$$
Q_{T}(f)=\langle T f, f\rangle \quad(f \in \mathcal{H})
$$

If $M$ is a closed linear subspace of $\mathcal{H}$ and $P$ the orthogonal projection of $\mathcal{H}$ onto $\mathcal{M}$, then the restriction $T_{\mathcal{M}}$ of the operator $P T$ to $\mathcal{M}$ is called the compression of $T$ to $\mathcal{M}$. It is easy to check that the quadratic form of $T_{\mathcal{M}}$ is just the restriction of $Q_{T}$ to $\mathcal{M}$, and so $W\left(T_{\mathcal{M}}\right) \subset W(T)$. It is an easy step from this to conclude that the convexity of the numerical range for any Hilbert space operator follows from the two dimensional case.

2.4. The two dimensional case. There are only a few possibilities for the numerical range of a linear operator on a two dimensional Hilbert space. One of these is trivial; if the operator is a scalar multiple of the identity then its numerical range is a single point (that scalar). So assume $T$ is not a scalar multiple of the identity on $\mathcal{H}$. Then there are only three possibilities:

(a) $T$ has just one eigenvalue. Then $W(T)$ is a closed disc centered at that eigenvalue.

(b) $T$ is normal. Then $T$ has two distinct eigenvalues (else it is a scalar multiple of the identity), and $W(T)$ is the closed line segment joining these eigenvalues.

(c) $T$ is not normal and has two distinct eigenvalues. Then $W(T)$ is a closed elliptical disc with foci at the eigenvalues. Case (a) can be viewed as the limiting case of this, where the foci coincide.

The last case will be particularly important for us. If a non-normal $T$ has distinct eigenvalues $\lambda_{1}$ and $\lambda_{2}$ then the dimensions of the elliptical disc $W(T)$ are determined by the angle between the eigenvectors. More precisely, let $f_{j}$ be an eigenvector for $\lambda_{j}$ with $\left\|f_{j}\right\|=1$ $(j=1,2)$, and set $\gamma=\left|\left\langle f_{1}, f_{2}\right\rangle\right|$. Then the major axis of $W(T)$ has length

$$
\left|\lambda_{1}-\lambda_{2}\right| / \sqrt{1-\gamma^{2}} \text {, }
$$

and the minor axis has length $\gamma$ times that of the major axis [18, page 113]. In particular, if $T$ has matrix

$$
\left[\begin{array}{cc}
\lambda_{1} & a \\
0 & \lambda_{2}
\end{array}\right]
$$


with respect to an orthonormal basis of $\mathbb{C}^{2}$, then the length of the major axis of $W(T)$ is $\sqrt{|a|^{2}+\left|\lambda_{1}-\lambda_{2}\right|^{2}}$, while that of the minor axis is just $|a|$ (see [15, Chapter 1] or [21, Chapter 1] for more details).

In studying composition operators, the case where the inducing function is a constantwhereupon the operator itself has rank one - is usually of little interest. The same is true here, but disposing of this simple case takes a little more work than one might expect. We do this right now (cf. [26, Theorem 2.4]).

2.5. Proposition. Suppose $T$ is a bounded linear operator on a Hilbert space of dimension $\geq 2$, and suppose the range of $T$ is one dimensional, so that there exist nonzero vectors $g$ and $h$ with $\|h\|=1$ such that

$$
T f=\langle f, g\rangle h \quad(f \in \mathcal{H}) .
$$

(a) If $g=$ ch for some $c \in \mathbb{C} \backslash\{0\}$ then $W(T)$ is the closed line segment from 0 to $\bar{c}$.

(b) If $g \perp h$ then $W(T)$ is the closed disc centered at the origin, of radius $\|g\| / 2$

(c) Otherwise $W(T)$ is a closed elliptical disc with foci at 0 and $\langle h, g\rangle$.

Proof. Since $\mathcal{H}$ has dimension $>1$ it contains a nonzero vector orthogonal to $g$, and $T$ therefore annihilates this vector, hence $0 \in W(T)$.

Let $\mathcal{M}$ be the linear span of $g$ and $h$, and $P$ the orthogonal projection of $\mathcal{H}$ onto $\mathcal{M}$. Then an easy calculation shows that

$$
\langle T f, f\rangle=\langle P T P f, f\rangle \quad(f \in \mathcal{H}),
$$

so that $\mathrm{W}(\mathrm{T})=\mathrm{W}(\mathrm{PTP})$. Now PTP is the orthogonal direct sum of the compression $T_{\mathcal{M}}$ of $T$ to $\mathcal{M}$, operating on $\mathcal{M}$, and the zero-operator acting on $\mathcal{M}^{\perp}$. Thus $W(P T P)$ is the convex hull of 0 and $W\left(T_{\mathcal{M}}\right)$, which equals $W\left(T_{\mathcal{M}}\right)$ because $0 \in W(T)$. Thus $W(T)=W\left(T_{\mathcal{M}}\right)$, so we may without loss of generality assume that $\mathcal{H}=\mathcal{M}$ and $T=T_{\mathcal{M}}$, which we do for the rest of the proof.

If $g=c h$ for some nonzero scalar $c$ we have

$$
\langle T f, f\rangle=\bar{c}\langle f, h\rangle\langle h, f\rangle=\bar{c}|\langle f, h\rangle|^{2}
$$

from which it follows easily that $W(T)$ is the closed line segment from the origin to $\bar{c}$.

If $g$ is not a scalar multiple of $h$ then set $e_{1}=h$ and

$$
e_{2}=a(g-\langle g, h\rangle h), \quad \text { where } \quad a^{-1}=\|g-\langle g, h\rangle h\| .
$$

Then the pair $\left(e_{1}, e_{2}\right)$ is an orthonormal basis for $\mathcal{H}$ relative to which $T$ has matrix

$$
A=\left[\begin{array}{cc}
\langle h, g\rangle & b \\
0 & 0
\end{array}\right], \quad \text { where } \quad b=a\left(\|g\|^{2}-|\langle g, h\rangle|^{2}\right) .
$$

The point here is that $b \neq 0$ because $g$ is not a scalar multiple of $h$. Thus if $g \perp h$ then $A=\left[\begin{array}{cc}0 & \|g\| \\ 0 & 0\end{array}\right]$, which has numerical range equal to the closed disc with center at the origin and radius $\|g\| / 2$; otherwise $W(A)$ is a closed elliptical disc with foci at 0 and $\langle h, g\rangle$. 
2.6. Corollary. Suppose $\varphi \equiv a \in \mathbb{U}$. If $a=0$ then $W\left(C_{\varphi}\right)$ is the closed interval $[0,1]$, otherwise it is a closed elliptical disc with foci at 0 and 1.

Proof. We have for every $f \in H^{2}$ :

$$
C_{\varphi} f=f(a)=\left\langle f, K_{a}\right\rangle \cdot 1,
$$

where $K_{a}$ is the reproducing kernel of Section 2.2. The result follows upon applying the previous Proposition with $g=K_{a}$ and $h \equiv 1$.

2.7. Symmetry of $W\left(C_{\varphi}\right)$. The following observation will prove useful in Sections 6 and 7:

If $\varphi$ is real on the real axis (or, equivalently, has real Taylor coefficients at the origin), then $W\left(C_{\varphi}\right)$ is symmetric with respect to the real axis.

If $\varphi$ has real Taylor coefficients at 0 , then the matrix of $C_{\varphi}$ with respect to the basis $\left\{z^{n}\right\}$ of $H^{2}$ will have real entries. In this case, if $w=\left\langle C_{\varphi} f, f\right\rangle$ for some $f$ in the unit sphere of $H^{2}$, then $\bar{w}=\left\langle C_{\varphi} \widetilde{f}, \widetilde{f}\right\rangle$, where $\widetilde{f}(z)=\overline{f(\bar{z})}$ is also in the unit sphere. Thus $W\left(C_{\varphi}\right)$ will be symmetric relative to the real axis.

2.8. Conformal automorphisms and dilations. A conformal automorphism is a univalent holomorphic mapping of $\mathbb{U}$ onto itself. Each such map is linear fractional, and can be represented as a product $\omega \cdot \alpha_{p}$, where

$$
\alpha_{p}(z):=\frac{p-z}{1-\bar{p} z} \quad(z \in \mathbb{U})
$$

for some fixed $p \in \mathbb{U}$ and $\omega \in \partial \mathbb{U}$ [30, Theorem 12.6, page 255]. The special automorphism $\alpha_{p}$ is particularly convenient to use because it is self-inverse and exchanges the origin with the point $p$ (see [32, Chapter 0], for example).

A dilation (more precisely, an $r$-dilation) is a map of the form $\delta_{r}(z)=r z$, where $r \in \mathbb{U}$. We call $r$ the dilation parameter of $\delta_{r}$. If $r>0$ we call $\delta_{r}$ a positive dilation. Clearly the composition operator $C_{\delta_{r}}$ on $H^{2}$ is compact and normal, having for each non-negative integer $n$ the monomial $z^{n}$ as an eigenvector with eigenvalue $r^{n}$. Easy arguments show that $W\left(C_{\delta_{r}}\right)$ is:

(a) the semi-open interval $(0,1]$ if $r>0$,

(b) the closed interval $[r, 1]$ if $-1<r \leq 0$.

(c) the convex hull of a sequence that spirals monotonically towards the origin if $r$ is not real (hence a polygon containing the origin in its interior, see [26, Proposition 2.2]).

Case (a) shows that the origin need not always belong to the numerical range of a composition operator not the identity, and (b) shows that the origin can belong to $W\left(C_{\varphi}\right)$ but not its interior. For non-dilations fixing the origin, however, the situation is different, as we will see in Theorem 3.8.

A conformal dilation (more precisely, a conformal $r$-dilation) is a map that is conformally conjugate to an $r$-dilation, i.e., a map $\varphi=\alpha^{-1} \circ \delta_{r} \circ \alpha$, where $r \in \mathbb{U}$ and $\alpha$ is a conformal automorphism of $\mathbb{U}$. Each such map fixes the point $p=\alpha^{-1}(0) \in U$. Now as we have previously noted, $\alpha=\rho_{\omega} \circ \alpha_{p}$ for some $\omega \in \partial \mathbb{U}$, where $\alpha_{p}$ is the special (self-inverse) automorphism defined by (4), and $\rho_{\omega}$ is the rotation defined by $\rho_{\omega}(z) \equiv \omega z$. From this it follows easily that $\varphi=\alpha_{p} \circ \delta_{r} \circ \alpha_{p}$. 
Finally, it is worth noting that for $\varphi$ as in the last paragraph and $\eta \in \partial \mathbb{U}$, the map $\psi:=$ $\rho_{\eta} \circ \varphi \circ\left(\rho_{\eta}\right)^{-1}$ is a conformal $r$-dilation that fixes the point $\eta p$. Now $C_{\psi}=V^{-1} C_{\varphi} V$, where $V$ is the unitary composition operator induced on $H^{2}$ by $\rho_{\eta}$, hence given any conformal dilation we may rotationally conjugate its fixed point $p$ to any other point of the circle $|z|=|p|$ at the cost of a unitary equivalence at the composition operator level. Since unitary equivalence does not change the numerical range we may, if it is convenient, assume with no loss of generality that the fixed point lies on the unit interval.

We will see in Section 4 that the conformal dilations that do not fix the origin, although simple in form, play a pivotal role in the study of zero-inclusion for the numerical range.

2.9. Zero-Inclusion for Automorphic Composition Operators. Numerical ranges of composition operators induced by conformal automorphisms are the focus of the authors' paper [4], where it is shown such automorphic composition operators usually, but not always, have numerical ranges whose closures are discs centered at the origin. Conformal automorphisms of $\mathbb{U}$, which as we have already noted are always linear-fractional maps, are classified as follows.

Hyperbolic: exactly two fixed points, both on $\partial \mathbb{U}$.

Parabolic: exactly one fixed point on $\partial \mathbb{U}$, no other fixed point on the Riemann sphere.

Elliptic: Conformally conjugate to a rotation about the origin; two fixed points in the sphere, one in $\mathbb{U}$, one outside its closure.

By Theorem 3.1 of [4], if $\varphi$ is an automorphism of $\mathbb{U}$ that is either of hyperbolic or parabolic type, then the closure of $W\left(C_{\varphi}\right)$ is a disc centered at the origin, from which it follows that $0 \in \operatorname{int} W\left(C_{\varphi}\right)$. The situation for elliptic automorphisms is more interesting.

An elliptic automorphism $\varphi$ of $\mathbb{U}$ must have the form

$$
\varphi=\alpha_{p} \circ \rho_{\omega} \circ \alpha_{p}
$$

for some $p \in \mathbb{U}$ and some $\omega \in \partial \mathbb{U}$. Suppose that $\varphi$ is given by (5). If $\omega$ is not a root of unity, then by Theorem 4.1 of [4], the closure of $W\left(C_{\varphi}\right)$ is a disk centered at the origin so that, just as for the other automorphic composition operators, $W\left(C_{\varphi}\right)$ contains 0 in its interior. To answer the Zero-Inclusion Question when $\omega$ is a root of unity, we observe that for every nonnegative integer $n$, the map $\alpha_{p}^{n}$ will be an eigenvector for $C_{\varphi}$ with eigenvalue $\omega^{n}$. It follows, by convexity, that if $\omega$ is neither 1 nor -1 , then $0 \in \operatorname{int} W\left(C_{\varphi}\right)$. If $\omega=1$, then, of course, $\varphi$ is the identity mapping and $W\left(C_{\varphi}\right)=\{1\}$. If $\omega=-1$, then by Corollary 4.4 of [4], $W\left(C_{\varphi}\right)$ is a ellipse with foci -1 and 1 that is degenerate if and only if $p=0$ (in which case $\varphi(z)=-z$ ). Summarizing, we see that if $\varphi$ is an elliptic automorphism, then $0 \in W\left(C_{\varphi}\right)$ unless $\varphi(z)=z$, and $0 \in \operatorname{int} W\left(C_{\varphi}\right)$ except when $\varphi(z)=z$ or $\varphi(z)=-z$. Finally, note that when $\varphi(z)=-z$, then $W\left(C_{\varphi}\right)=[-1,1]$, which is consistent with our description of numerical ranges of composition operators induced by dilations.

2.10. The Denjoy-Wolff Theorem. Every holomorphic self-map $\varphi$ of the unit disc $\mathbb{U}$ that is not conformally conjugate to a rotation has a unique attractive fixed point $p$ in the closure of $\mathbb{U}$ which is called the Denjoy-Wolff point of $\varphi$. If $p \in \partial \mathbb{U}$, then $p$ is fixed in the sense that $\lim _{r \rightarrow 1^{-}} \varphi(r p)=p$. The Denjoy-Wolff point $p$ of $\varphi$ is globally attractive: for each $z \in \mathbb{U}$, the limit of the iterate sequence $\left(\varphi^{[n]}(z)\right)$ is $p$. Hence, in particular, a self-map of $\mathbb{U}$ that is not the identity may fix at most one point of $\mathbb{U}$, a fact we will use without comment 
in the sequel. For a proof and further discussion of the Denjoy-Wolff theorem, the reader may consult, e.g., [32, Chapter 5].

\section{Zero Containment: Part I}

Here we begin our investigation of when $0 \in W\left(C_{\varphi}\right)$. We commented earlier that for each $0<r<1$ the positive-dilation map $\varphi(z) \equiv r z$ gives rise to a normal composition operator whose numerical range is the half-open real interval $(0,1]$, so 0 is not always in $W\left(C_{\varphi}\right)$. On the other hand, if $\varphi$ is not univalent then $C_{\varphi}^{*}$ annihilates a difference of reproducing kernels, in which case $0 \in W\left(C_{\varphi}\right)$.

In this section we prove that for any composition operator not the identity, the origin lies in the closure of the numerical range. We prove that the origin lies in the interior of $W\left(C_{\varphi}\right)$ whenever $C_{\varphi}$ fails to have dense range (which happens, for example, whenever $\varphi$ is not univalent). Then we focus on holomorphic self-maps $\varphi$ of $\mathbb{U}$ that fix the origin, proving that $0 \in \operatorname{int} W\left(C_{\varphi}\right)$ unless $\varphi$ has the form $\varphi(z)=t z$ for some $t \in[-1,1]$. This result, together with our observations in Sections 2.8 and 2.9 concerning numerical ranges for composition operators induced by dilations and rotations, settles the zero inclusion issue for composition operators with symbols that fix the origin. We wait until Section 4 to take up the fascinating case of maps that fix points of $\mathbb{U}$ other than the origin.

We employ the notation $\bar{W}(T)$ for the closure of the numerical range of an operator $T$. Note that a composition operator is the identity map on $H^{2}$ if and only if its inducing function is the identity map on $\mathbb{U}$.

3.1. Theorem. If $\varphi$ is any holomorphic self-map of $\mathbb{U}$ that is not the identity map then $0 \in \bar{W}\left(C_{\varphi}\right)$.

Proof. For $a \in \mathbb{U}$ let $f_{a}=K_{a} /\left\|K_{a}\right\|$, where $K_{a}$ is the reproducing kernel discussed in Section 2.2 , and let

a point of $W\left(C_{\varphi}\right)$. Since

$$
w_{a}=\left\langle C_{\varphi} f_{a}, f_{a}\right\rangle=\frac{\left\langle C_{\varphi} K_{a}, K_{a}\right\rangle}{\left\|K_{a}\right\|^{2}}
$$

$$
\left\|K_{a}\right\|^{2}=\left\langle K_{a}, K_{a}\right\rangle=K_{a}(a)=\frac{1}{1-|a|^{2}},
$$

we have for each point $a \in \mathbb{U}$ :

$$
\begin{aligned}
w_{a} & =\left(1-|a|^{2}\right)\left\langle K_{a}, C_{\varphi}^{*} K_{a}\right\rangle=\left(1-|a|^{2}\right)\left\langle K_{a}, K_{\varphi(a)}\right\rangle \\
& =\left(1-|a|^{2}\right) K_{a}(\varphi(a))=\frac{1-|a|^{2}}{1-\bar{a} \varphi(a)} .
\end{aligned}
$$

Now because $\varphi$ is not the identity map on $\mathbb{U}$ its radial limit function is not almost-everywhere equal to the identity map on $\partial \mathbb{U}$, so there exists $\zeta \in \mathbb{U}$ for which the radial limit $\varphi(\zeta)$ exists and is not equal to $\zeta$. Thus, by the result of the last calculation, if $a \rightarrow \zeta$ then the points $w_{a}$ of $W\left(C_{\varphi}\right)$ converge to zero, hence $0 \in \bar{W}\left(C_{\varphi}\right)$, as desired.

Next we generalize the observation that $0 \in W\left(C_{\varphi}\right)$ whenever $\varphi$ is not univalent. The crucial idea is the notion of normal eigenvalue. 
3.2. Normal eigenvalues. Suppose $T$ is a bounded linear operator on Hilbert space, and $\lambda$ is an eigenvalue of $T$. We say $\lambda$ normal for $T$ if

$$
\operatorname{ker}(T-\lambda I)=\operatorname{ker}\left(T^{*}-\bar{\lambda} I\right) .
$$

This terminology reflects the elementary fact that the eigenvalues of a normal operator are normal eigenvalues.

By Theorem 2 of [20], every eigenvalue of an arbitrary Hilbert space operator $T$ that lies on the boundary of $W(T)$ must be a normal eigenvalue. We supply a proof different from the one in [20], which is based on the following.

3.3. Proposition. Suppose that $T$ is a bounded linear operator on a Hilbert space $\mathcal{H}$ and that $f$ is a unit vector in $\mathcal{H}$ such that $\langle T f, f\rangle$ belongs to the boundary of $W(T)$. Then $T^{*} f$ is a linear combination of $f$ and $T f$.

Proof. Suppose, in order to prove the contrapositive, that $T^{*} f$ is not a linear combination of $f$ and $T f$. Then there is a unit vector $g \in \mathcal{H}$ that is orthogonal to both $f$ and $T f$ yet $\left\langle g, T^{*} f\right\rangle \neq 0$. Let $\mathcal{M}$ denote the two-dimensional subspace of $\mathcal{H}$ with orthonormal basis $\{f, g\}$ and let $\lambda=\langle T f, f\rangle$. The compression of $T$ to $\mathcal{M}$, which we denote $T_{\mathcal{M}}$, has matrix

$$
\left[\begin{array}{cc}
\lambda & \langle T g, f\rangle \\
0 & \langle T g, g\rangle
\end{array}\right]
$$

relative to $\{f, g\}$. Because $\langle T g, f\rangle=\left\langle g, T^{*} f\right\rangle$ is nonzero, the numerical range of $T_{\mathcal{M}}$ is a nondegenerate ellipse with foci $\lambda$ and $\langle T g, g\rangle$. In particular, $\lambda$ lies in the interior of $W\left(T_{\mathcal{M}}\right) \subseteq W(T)$.

3.4. Corollary. Suppose that $T$ is a bounded operator on a Hilbert space $\mathcal{H}$; then every eigenvalue of $T$ that lies on the boundary of $W(T)$ is a normal eigenvalue.

Proof. Suppose $f$ is a unit vector in $\mathcal{H}$ and $\lambda \in \partial W(T)$ satisfies $T f=\lambda f$. By Proposition 3.3, $T^{*} f=\alpha f$ for some scalar $\alpha$. We have $\alpha=\left\langle T^{*} f, f\right\rangle=\langle f, T f\rangle=\bar{\lambda}$, as desired. Because $\bar{\lambda}$ belongs to $\partial W\left(T^{*}\right)$ whenever $\lambda$ belongs to $\partial W(T)$, the same argument shows $\operatorname{ker}\left(T^{*}-\bar{\lambda} I\right) \subseteq \operatorname{ker}(T-\lambda I)$.

The preceding corollary shows that 0 belongs to the interior of $W(T)$ whenever $T$ is injective and fails to have dense range. We present the argument in the context of composition operators.

3.5. Corollary. Suppose $\varphi$ is a nonconstant holomorphic self-map of $\mathbb{U}$. If the polynomials in $\varphi$ are not dense in $H^{2}$, then $0 \in \operatorname{int} W\left(C_{\varphi}\right)$.

Proof. Because $\varphi$ is nonconstant, $C_{\varphi}$ is one-to-one. The hypothesis on $\varphi$ means that the range of $C_{\varphi}$ is not dense, hence its orthogonal complement, the kernel of $C_{\varphi}^{*}$, is not zero. Thus zero is an eigenvalue of $C_{\varphi}^{*}$, and therefore belongs to $W\left(C_{\varphi}^{*}\right)$. But zero is not a normal eigenvalue, so by Theorem 3.4 it cannot be a boundary point of $W\left(C_{\varphi}^{*}\right)$. Thus, because the numerical range of the adjoint operator is the complex conjugate of the original numerical range, 0 lies in the interior of $W\left(C_{\varphi}\right)$. 
3.6. Corollary. Suppose $\varphi$ is a nonconstant holomorphic self-map of $\mathbb{U}$. If $\varphi$ is not oneto-one then $0 \in \operatorname{int} W\left(C_{\varphi}\right)$.

Proof. As we noted earlier, if $\varphi$ is not one-to-one then $C_{\varphi}^{*}$ annihilates a nontrivial difference of reproducing kernels, so the kernel of $C_{\varphi}^{*}$ is not $\{0\}$. Thus the closure of the range of $C_{\varphi}$, which is $\left(\operatorname{ker} C_{\varphi}^{*}\right)^{\perp}$, is not all of $H^{2}$, i.e., $\operatorname{ran} C_{\varphi}$ is not dense. Thus, by Corollary 3.5, $0 \in \operatorname{int} W\left(C_{\varphi}\right)$.

3.7. Remark. The work of Section 2.6 disposes of the case $\varphi \equiv$ constant; if the constant is nonzero then zero is in the interior of $W\left(C_{\varphi}\right)$, otherwise $W\left(C_{\varphi}\right)$ is the closed unit interval.

We now consider the question of zero-inclusion for composition operators induced by maps $\varphi$ that fix the origin. If $\varphi$ is a dilation or rotation, then work of Sections 2.8 and 2.9 yields complete information about zero inclusion; in particular, $0 \in \operatorname{int} W\left(C_{\varphi}\right)$ unless $\varphi$ belongs to the family $\{z \mapsto t z: t \in[-1,1]\}$. We now prove that this family provides the only exception to the interior zero-inclusion rule among all holomorphic self-maps of $\mathbb{U}$ that fix the origin.

3.8. Theorem. Suppose that $\varphi$ is a holomorphic self-map of $\mathbb{U}$ fixing the origin and that $\varphi$ does not have the form $\varphi(z)=t z$ for some $t \in[-1,1]$. Then $0 \in \operatorname{int} W\left(C_{\varphi}\right)$.

Proof. $\varphi$ not constant, for otherwise it would be identically equal to zero (since $\varphi(0)=0$ ), and hence would have the forbidden form $\varphi(z)=t z$ with $t=0$. Let $\lambda=\varphi^{\prime}(0)$. We may assume that $\lambda \neq 0$ for otherwise $\varphi$ is not univalent (and nonconstant) and the desired conclusion follows from Corollary 3.6. We may also assume that $\varphi$ is neither a rotation or dilation (by the work of Sections 2.8 and 2.9, as discussed above). Thus $\varphi$ 's MacLaurin expansion can be written in the form

$$
\varphi(z)=\lambda z\left[1+b z^{m}(1+h(z))\right]
$$

where $m$ is a positive integer, $b \neq 0$, and $h$ is holomorphic in $\mathbb{U}$ with $h(0)=0$. The binomial theorem then shows that for each positive integer $n$,

$$
\varphi(z)^{n}=\lambda^{n} z^{n}+n b \lambda^{n} z^{n+m}+\text { terms of higher order in } z .
$$

Now the matrix of $C_{\varphi}$ with respect to the orthonormal basis $\left\{z^{k}\right\}_{0}^{\infty}$ has as its $n$-th column the sequence of power series coefficients of $\varphi^{n}$, and any compression of $C_{\varphi}$ to the subspace spanned by a subset of these monomials has as its matrix the corresponding principal submatrix. We are going to focus on the linear $\operatorname{span} M_{n}$ of $\left\{z^{n}, z^{m+n}\right\}$; because $m>0$ this subspace is two dimensional. Let $T_{n}$ be the compression of $C_{\varphi}$ to $M_{n}$, so matrix of $T_{n}$ with respect to the orthonormal basis $\left\{z^{n}, z^{n+m}\right\}$ is

$$
\begin{aligned}
B_{n} & :=\left[\begin{array}{cc}
\widehat{\varphi^{n}}(n) & 0 \\
\widehat{\varphi^{n}}(n+m) & \widehat{\varphi^{n+m}}(n+m)
\end{array}\right] \\
& =\left[\begin{array}{cc}
\lambda^{n} & 0 \\
n b \lambda^{n} & \lambda^{n+m}
\end{array}\right] \\
& =\lambda^{n} A_{n},
\end{aligned}
$$


where

$$
A_{n}=\left[\begin{array}{cc}
1 & 0 \\
n b & \lambda^{m}
\end{array}\right]
$$

It is enough to show that $0 \in \operatorname{int} W\left(A_{n}\right)$ for large enough $n$. By the discussion of Section 2.4 we know that $W\left(A_{n}\right)$ is a closed elliptical disc with foci at the points 1 and $\lambda^{m}$ and minor axis of length $n|b|$. For $n$ large enough the length of the minor semi-axis of $W\left(A_{n}\right)$ will exceed the modulus of its center, thus placing the origin in the interior; this happens whenever $n>\left|1+\lambda^{m}\right| /|b|$.

The preceding Theorem completes our solution to the Zero-Inclusion Question for composition operators induced by mappings $\varphi$ that fix the origin: 0 does not belong to $W\left(C_{\varphi}\right)$ if and only if $\varphi(z)=t z$ for some $t \in(0,1]$, and $0 \in W\left(C_{\varphi}\right)$ implies $0 \in \operatorname{int} W\left(C_{\varphi}\right)$ unless $\varphi$ has the form $\varphi(z)=t z$ for some $t \in[-1,0]$. In the next section, we consider the much more subtle situation that arises when $\varphi$ fixes a non-zero point of $\mathbb{U}$.

\section{Zero Containment: Part II}

Our goal in this section is to show that if $\varphi$ fixes a nonzero point in $\mathbb{U}$ and is neither the identity map nor a positive conformal dilation in the sense of Section 2.8 , then $0 \in$ int $W\left(C_{\varphi}\right)$. For the moment, however, we assume only that $\varphi$ is a holomorphic self-map of $\mathbb{U}$ that fixes a point $p \in \mathbb{U} \backslash\{0\}$, and that $\varphi$ is not an automorphism (the zero-containment story for automorphisms has already been told in Section 2.9). As in the proof of Theorem 3.8 we seek to compute appropriate two by two principal submatrices of the matrix of $C_{\varphi}$, but instead of computing this matrix relative to the monomial basis $\left(z^{n}\right)_{0}^{\infty}$ of $H^{2}$ we use instead Guyker's basis. This is the sequence of functions $\left(b_{n}\right)_{0}^{\infty}$ defined for $z \in \mathbb{U}$ by:

$$
b_{n}(z):=\frac{\sqrt{1-|p|^{2}}}{1-\bar{p} z}\left(\frac{z-p}{1-\bar{p} z}\right)^{n}=\frac{K_{p}}{\left\|K_{p}\right\|}\left(-\alpha_{p}(z)\right)^{n}
$$

where $K_{p}$ is the reproducing kernel for $p$ (as defined in Section 2.2) and $\alpha_{p}$ is the special automorphism defined by (4).

In [16] Guyker established that $\left(b_{n}\right)_{0}^{\infty}$ is an orthonormal basis for $H^{2}$ relative to which the matrix of $C_{\varphi}$ is lower triangular, with the derivative-power $\varphi^{\prime}(p)^{n}$ as the $n$-th element of the main diagonal $(n=0,1,2, \ldots)$. Guyker used his basis to study the reducing subspaces of $C_{\varphi}$. Actually Guyker's basis is a special case of an orthonormal basis used by F. Malmquist in 1928 to study interpolation problems in $H^{2}$ ([25], see also [35, §10.7, pp. 304-309]). We are indebted to Professor Harold S. Shapiro for pointing out these references.

Here we focus on the compression of $C_{\varphi}$ to the two dimensional subspace

$$
\mathcal{M}_{n, k}:=\operatorname{span}\left\{b_{n}, b_{n+k}\right\} \quad(n \geq 0, k \geq 1) .
$$

Relative to the orthonormal basis $\left(b_{n}, b_{n+k}\right)$ this compression has matrix

$$
A_{n, k}:=\left[\begin{array}{cc}
\varphi^{\prime}(p)^{n} & 0 \\
\left\langle C_{\varphi} b_{n}, b_{n+k}\right\rangle & \varphi^{\prime}(p)^{n+k}
\end{array}\right],
$$

so, upon noting that the Schwarz-Pick Lemma insures that $\left|\varphi^{\prime}(p)\right|<1$ and proceeding as in the proof of Theorem 3.8, we can establish that $0 \in \operatorname{int} W\left(C_{\varphi}\right)$ by showing that for some 
fixed positive integer $k$ :

$$
\lim _{n \rightarrow \infty} \frac{\left\langle C_{\varphi} b_{n}, b_{n+k}\right\rangle}{\left|\varphi^{\prime}(p)^{n}\right|}=\infty
$$

Without further ado, let us compute the crucial inner product entry.

4.1. Lemma. Suppose $\varphi$ is a holomorphic self-map of $\mathbb{U}$ that fixes a point $p \in \mathbb{U}$. Let $\left(b_{n}\right)$ be the Guyker basis for the point $p$. Then for each non-negative integer $n$ and each positive integer $k$,

$$
\left\langle C_{\varphi} b_{n}, b_{n+k}\right\rangle=\frac{1-|p|^{2}}{k !} H_{n, k}^{(k)}(p)
$$

where

$$
H_{n, k}(z)=\frac{(1-\bar{p} z)^{n+k}}{(1-\bar{p} \varphi(z))^{n+1}}\left(\frac{\varphi(z)-p}{z-p}\right)^{n}
$$

Proof. We begin by writing the $H^{2}$ inner product in question as a complex contour integral

$$
\left\langle C_{\varphi} b_{n}, b_{n+k}\right\rangle=\frac{1}{2 \pi i} \int_{\partial \mathbb{U}} b_{n}(\varphi(z)) \overline{b_{n+k}(z)} \frac{d z}{z},
$$

where $\partial \mathbb{U}$ is positively oriented. We compute the integrand by noticing that $\left|\alpha_{p}(z)\right|=1$ when $|z|=1$, so that the complex conjugate of $\alpha_{p}(z)$ is its reciprocal. This observation, along with some algebraic manipulation yields, for $|z|=1$,

hence

$$
b_{n}(\varphi(z)) \overline{b_{n+k}(z)}=\left(1-|p|^{2}\right) H_{n, k}(z) \frac{z}{(z-p)^{k+1}},
$$

$$
\left\langle C_{\varphi} b_{n}, b_{n+k}\right\rangle=\frac{1-|p|^{2}}{2 \pi i} \int_{\partial \mathbb{U}} H_{n, k}(z) \frac{d z}{(z-p)^{k+1}}=\frac{\left(1-|p|^{2}\right)}{k !} H_{n, k}^{(k)}(p),
$$

as desired.

When $k=1$, the preceding Lemma yields the following.

4.2. Corollary. Under the hypotheses of Lemma 4.1 we have for each non-negative integer $n$,

$$
\left\langle C_{\varphi} b_{n}, b_{n+1}\right\rangle=\varphi^{\prime}(p)^{n-1}\left[n\left(1-|p|^{2}\right) \frac{\varphi^{\prime \prime}(p)}{2}-(n+1) \bar{p} \varphi^{\prime}(p)\left(1-\varphi^{\prime}(p)\right)\right] .
$$

This last corollary allows some preliminary observations that focus attention on the heart of our problem. We have already seen that that the desired "interior zero-containment" happens whenever $\varphi$ is not univalent (Theorem 3.6). The next result, part of which also appears in [26, Section 3], shows that interior zero-containment also happens whenever the derivative of $\varphi$ at the fixed point is non-positive. 
4.3. Proposition. Suppose $\varphi$ is a holomorphic self-map of $\mathbb{U}$ that is univalent but not the identity, and fixes a non-zero point $p \in \mathbb{U}$. If $\varphi^{\prime}(p)$ is not strictly positive then $0 \in$ int $W\left(C_{\varphi}\right)$.

Proof. Univalence forces $\varphi^{\prime}(p) \neq 0$, which we assume from now on. The form of the matrix of $C_{\varphi}$ with respect to $\left(b_{n}\right)_{0}^{\infty}$ shows that the complex conjugate of each number $\varphi^{\prime}(p)^{n}$ is an eigenvalue of $C_{\varphi}^{*}$ (see, e.g., [32, Section 6.2] for the details), and therefore lies in the numerical range of that operator. Thus $\varphi^{\prime}(p)^{n} \in W\left(C_{\varphi}\right)$ for each $n$.

Suppose that $\varphi^{\prime}(p)$ is non-real. Then as $n \rightarrow \infty$ the sequence $\left(\varphi^{\prime}(p)^{n}\right)$ spirals in towards the origin, circling it infinitely often, thus (by the Toeplitz-Hausdorff Theorem) placing the origin in the interior of $W\left(C_{\varphi}\right)$.

The only remaining possibility is $\varphi^{\prime}(p)<0$, for which the argument just given at least locates the origin in $W\left(C_{\varphi}\right)$. To see that it is in the interior one need only note that by the case $n=0$ of Corollary 4.2 ,

$$
\left\langle C_{\varphi} b_{0}, b_{1}\right\rangle=\bar{p}\left(\varphi^{\prime}(p)-1\right) \neq 0 .
$$

Thus the numerical range of the matrix

$$
\left[\begin{array}{cc}
1 & 0 \\
\left\langle C_{\varphi} b_{0}, b_{1}\right\rangle & \varphi^{\prime}(p)
\end{array}\right]
$$

is a nondegenerate ellipse with foci at 1 and $\varphi^{\prime}(p)$. Since $\varphi^{\prime}(p)<0$, this ellipse has the origin in its interior, and because the matrix represents a compression of $C_{\varphi}$ (to the subspace spanned by $b_{0}$ and $\left.b_{1}\right)$, the origin lies in the interior of $W\left(C_{\varphi}\right)$ as well.

According to the discussion above, if $\varphi$ fixes a point $p \in U \backslash\{0\}$, then the containment of 0 in the interior of $W\left(C_{\varphi}\right)$ is in doubt only when $\varphi$ is univalent and $\varphi^{\prime}(p)>0$; for example, when $\varphi$ is a positive conformal dilation (terminology as in Section 2.8). The next resultthe principal one of this section-reduces the zero-containment issue to precisely the class of positive conformal dilations.

4.4. Theorem. Suppose $\varphi$ is a holomorphic self-map of $\mathbb{U}$ that fixes a non-zero point $p \in \mathbb{U}$ and is neither the identity map on $\mathbb{U}$ nor a positive conformal dilation. Then $0 \in \operatorname{int} W\left(C_{\varphi}\right)$.

Proof. By Corollary 3.6 we may assume that $\varphi$ is univalent, and by the work of Section 2.9, we may assume that $\varphi$ is not an elliptic automorphism. By Proposition 4.3 we may require further that $\varphi$ not be any sort of conformal dilation. Note that for integers $n \geq 0$ and $k>0$ the function $H_{n, k}$ that is the major player in Lemma 4.1 can be written as

$$
H_{n, k}=L^{n+1} M^{k-1} Q^{n}
$$

where $L, M$, and $Q$ are holomorphic functions on $\mathbb{U}$ defined by:

$$
L(z)=\frac{1-\bar{p} z}{1-\bar{p} \varphi(z)}, M(z)=1-\bar{p} z, \text { and } Q(z)=\frac{\varphi(z)-p}{z-p}
$$

( $Q$ being holomorphic at $p$, with $Q(p)=\varphi^{\prime}(p)$, because $\varphi(p)=p$ ).

Clearly $L$ is non-vanishing on $\mathbb{U}$, and the univalence of $\varphi$ insures that the same is true of $Q$. Note also that

$$
L(p)=1, \quad M(p)=1-|p|^{2}, \quad \text { and } \quad Q(p)=\varphi^{\prime}(p),
$$


so that

$$
H_{n, k}(p)=\left(1-|p|^{2}\right)^{k-1} \varphi^{\prime}(p)^{n}=\text { const. } \varphi^{\prime}(p)^{n},
$$

where "const." does not depend on $n$.

Recall that our goal is to establish (7) for some fixed $k>0$, so by Lemma 4.1 it is enough to show that

$$
\lim _{n \rightarrow \infty} \frac{H_{n, k}^{(k)}(p)}{H_{n, k}(p)}=\infty .
$$

To motivate what is to follow we first consider the case $k=1$, and for convenience write $H_{n, 1}$ simply as $H_{n}$. Thus $H_{n}=L^{n+1} Q^{n}$, whereupon logarithmic differentiation yields

$$
\frac{H_{n}^{\prime}}{H_{n}}=(n+1) \frac{L^{\prime}}{L}+n \frac{Q^{\prime}}{Q}=n\left[\frac{L^{\prime}}{L}+\frac{Q^{\prime}}{Q}\right]+\frac{L^{\prime}}{L},
$$

which we rewrite as:

$$
\frac{H_{n}^{\prime}}{H_{n}}=n F+B, \quad \text { where } \quad F:=\frac{L^{\prime}}{L}+\frac{Q^{\prime}}{Q} \quad \text { and } \quad B:=\frac{L^{\prime}}{L} .
$$

Note that $F$ and $B$ are holomorphic on $\mathbb{U}$ because neither $L$ nor $Q$ vanishes anywhere on $\mathbb{U}$. Moreover (11) shows that if $F(p) \neq 0$ then (10) holds for $k=1$, hence

$$
F(p) \neq 0 \Longrightarrow 0 \in \operatorname{int} W\left(C_{\varphi}\right) .
$$

Although we will not need this in the sequel, it is interesting to note that

$$
F(p) \neq 0 \Longleftrightarrow\left(1-|p|^{2}\right) \frac{\varphi^{\prime \prime}(p)}{2} \neq \bar{p} \varphi^{\prime}(p)\left(1-\varphi^{\prime}(p)\right),
$$

so the reader who is tiring of the zero-containment issue can rest assured that for at least all the maps $\varphi$ satisfying the condition above, $0 \in \operatorname{int} W\left(C_{\varphi}\right)$.

For those who wish to continue, the crucial point is that (relaxing for the moment our requirement that $\varphi$ not be a conformal dilation)

$$
F \equiv 0 \text { on } \mathbb{U} \Longleftrightarrow \varphi \text { is a conformal dilation with fixed point at } p \text {. }
$$

This follows from the fact that $F$ is the logarithmic derivative of $L Q=\left(\alpha_{p} \circ \varphi\right) / \alpha_{p} \quad($ from $(9))$, hence $F \equiv 0$ if and only if $\alpha_{p} \circ \varphi$ is a constant multiple of $\alpha_{p}$, i.e., if and only if $\varphi$ is a conformal dilation.

Since we have already done the case $F(p) \neq 0$ let's suppose that $F(p)=0$. Because we are demanding that $\varphi$ not be a conformal dilation, $F$ is not identically zero on $\mathbb{U}$, and since $F$ is holomorphic on $\mathbb{U}$ this guarantees an integer $k \geq 2$ such that

$$
F(p)=F^{\prime}(p)=\cdots=F^{(k-2)}(p)=0, \quad \text { but } \quad F^{(k-1)}(p) \neq 0 .
$$

It is for this integer $k$ that we are going to prove (10).

From (11) we see that for each integer $j \geq 0$ :

$$
\left(\frac{H_{n}^{\prime}}{H_{n}}\right)^{(j)}=n F^{(j)}+B^{(j)},
$$


so that the numerical sequence $\left(\left(H_{n}^{\prime} / H_{n}\right)^{(j)}(p): n \geq 0\right)$ is bounded for $0 \leq j \leq k-2$, and it $\rightarrow \infty$ for $j=k-1$. Using

and the product rule, we obtain

$$
H_{n}^{(k)}=\left(H_{n} \cdot \frac{H_{n}^{\prime}}{H_{n}}\right)^{(k-1)}
$$

$$
\frac{H_{n}^{(k)}(p)}{H_{n}(p)}=\left(\frac{H_{n}^{\prime}}{H_{n}}\right)^{(k-1)}(p)+\sum_{j=0}^{k-2}\left(\begin{array}{c}
k-1 \\
j
\end{array}\right) \frac{H_{n}^{(k-1-j)}(p)}{H_{n}(p)}\left(\frac{H_{n}^{\prime}}{H_{n}}\right)^{(j)}(p) .
$$

Because $\left(H_{n}^{\prime} / H_{n}\right)^{(k-1)}(p) \rightarrow \infty$ as $n \rightarrow \infty$ the same will be true of $H_{n}^{(k)}(p) / H_{n}(p)$ provided the sum on the right of (13) remains bounded as $n \rightarrow \infty$. We already have observed that the terms in the sum of the form $\left(H_{n}^{\prime} / H_{n}\right)^{(j)}(p)$ are bounded independent of $n$ (given the range of the index $j$ ). The other terms in the sum take the form $H_{n}^{(J)}(p) / H_{n}(p)$, where $J$ varies from 1 to $k-1$. To see that the sequence $\left(H_{n}^{(J)}(p) / H_{n}(p)\right)$ is bounded (in $n$ ) for this range of index $J$, apply an inductive argument. The boundedness of $\left(H_{n}^{(1)}(p) / H_{n}(p)\right)$ has already been noted $(j=0$ in $(12))$. That the boundedness of $\left(H_{n}^{(v)}(p) / H_{n}(p)\right)$ for $v=1, \ldots, J-1$ implies that of $\left(H_{n}^{(J)}(p) / H_{n}(p)\right)$, when $1<J \leq k-1$, follows from (13): simply replace $k$ with $J$. We have established

$$
\lim _{n \rightarrow \infty} \frac{H_{n}^{(k)}(p)}{H_{n}(p)}=\infty
$$

which is almost the desired result. To finish the job note that $H_{n, k}=H_{n} M_{k}$, where

$$
M_{k}(z)=M(z)^{k-1}=(1-\bar{p} z)^{k-1} \quad(z \in \mathbb{U}) .
$$

Thus

$$
H_{n, k}^{(k)}=H_{n}^{(k)} M_{k}+\sum_{j=0}^{k-1}\left(\begin{array}{c}
k \\
j
\end{array}\right) H_{n}^{(j)} M_{k}^{(k-j)}
$$

so that

$$
\frac{H_{n, k}^{(k)}(p)}{H_{n, k}(p)}=\frac{H_{n}^{(k)}(p)}{H_{n}(p)}+\sum_{j=0}^{k-1} c_{j} \frac{H_{n}^{(j)}(p)}{H_{n}(p)},
$$

where on the right-hand side, each $c_{j}$ is a non-zero constant that does not depend on $n$. We have previously shown that for each $0 \leq j \leq k-1$ the sequence

$$
\left(\frac{H_{n}^{(j)}(p)}{H_{n}(p)}: n \geq 0\right)
$$

is bounded, and that it $\rightarrow \infty$ for $j=k$. Thus the left-hand side of (15) $\rightarrow \infty$ as $n \rightarrow \infty$, as we wished to show. 


\section{Zero Containment: Part III}

The work of Sections 3 and 4 has established that if $\varphi$ is a holomorphic self-map of $\mathbb{U}$ that fixes a point $p \in \mathbb{U}$ and neither the identity nor a positive conformal dilation, then $0 \in W\left(C_{\varphi}\right)$. What happens when $\varphi$ is a positive conformal dilation? We have already observed (Section 2.8) that $0 \notin W\left(C_{\varphi}\right)$ whenever $\varphi$ is a positive conformal dilation that fixes the origin (i.e. an "ordinary" positive dilation). However the next result shows that zero-containment reappears whenever the fixed point is located sufficiently far from the origin.

5.1. Theorem. Suppose $\varphi$ is a positive conformal dilation that fixes the point $p \in \mathbb{U}$ and has dilation parameter $r(0<r<1)$. Then the following three conditions are equivalent:

(a) $0 \in W\left(C_{\varphi}\right)$.

(b) $0 \in \operatorname{int} W\left(C_{\varphi}\right)$.

(c) $|p|>\sqrt{r}$.

Moreover if $|p| \leq \sqrt{r}$ then $\operatorname{Re} C_{\varphi}:=\frac{1}{2}\left(C_{\varphi}+C_{\varphi}^{*}\right)$ is strictly positive-definite on $H^{2}$.

Proof. By the work of Section 2.8 we know that $\varphi(z)=\alpha_{p}\left(r \alpha_{p}(z)\right)$ for $z \in \mathbb{U}$, where $r \in(0,1)$ is the dilation parameter of $\varphi$.

$(\mathrm{c}) \rightarrow(\mathrm{b})$ : Suppose $|p|>r$. We know that $C_{\varphi}\left(\alpha_{p}^{n}\right)=r^{n} \alpha_{p}^{n}$ for each non-negative integer $n$. For each positive integer $k$ let $\mathcal{M}_{k}$ be the two dimensional $C_{\varphi}$-invariant subspace spanned by 1 and $\alpha_{p}^{k}$, and let $A_{k}$ be the restriction of $C_{\varphi}$ to $\mathcal{M}_{k}$. By the discussion of Section 2.4 we know that $W\left(A_{k}\right)$ is a nondegenerate ellipse with foci at 1 and $r^{k}$, with major axis of length

$$
\ell=\frac{1-r^{k}}{\sqrt{1-\gamma_{k}^{2}}} \quad \text { where } \quad \gamma_{k}:=\left|\left\langle\alpha_{p}^{k}, 1\right\rangle\right|=\left|\alpha_{p}^{k}(0)\right|=|p|^{k}
$$

(the nondegeneracy comes from the fact that the vectors 1 and $\alpha_{p}^{k}$ are not orthogonal in $H^{2}$, as the above calculation shows). The center $c$ of the ellipse is the midpoint of the foci, i.e., $c=\left(1+r^{k}\right) / 2$ on the positive real axis. Now $0 \in \operatorname{int} W\left(A_{k}\right)$ if and only if $c<\ell / 2$, i.e., if and only if

$$
1+r^{k}<\frac{1-r^{k}}{\sqrt{1-|p|^{2 k}}}
$$

Some algebraic manipulation shows this last condition to be equivalent to

$$
|p|>\left(\frac{2}{1+r^{k}}\right)^{1 / k} \sqrt{r}
$$

which, because we are assuming $|p|>\sqrt{r}$, is satisfied for all sufficiently large $k$. For any such $k$

as we wished to show.

$$
0 \in \operatorname{int} W\left(A_{k}\right) \subset \operatorname{int} W\left(C_{\varphi}\right)
$$

(b) $\rightarrow$ (a): Trivial.

(a) $\rightarrow$ (c): Suppose $|p| \leq \sqrt{r}$. We will prove the strict positivity of $\operatorname{Re} C_{\varphi}$, which implies that $0 \notin W\left(C_{\varphi}\right)$. 
By the discussion of Section 2.8 we may, without loss of generality, assume that $0<p<1$. Then a little calculation proceeding from the formula $\varphi(z)=\alpha_{p}\left(r \alpha_{p}(z)\right)$ shows that

$$
\varphi(z)=\frac{a z+1}{d-z}
$$

where

$$
a=\frac{r-p^{2}}{p(1-r)} \quad \text { and } \quad d=\frac{1-p^{2} r}{p(1-r)}
$$

(the assumption that $p$ is real simply avoids some complex conjugates that would otherwise have appeared in these last two formulas).

Clearly $d>0$; furthermore the hypothesis $p \leq \sqrt{r}$ implies that $a \geq 0$, with equality if and only if $p=\sqrt{r}$. In this interesting transitional case, about which we will say more later, $\varphi(z) \equiv(d-z)^{-1}$ with $d=(1+r) / \sqrt{r}$.

Our hypothesis relating $p$ and $r$ also implies that $d \geq(1+r) / \sqrt{r}>2$, from which it follows that $\varphi$ takes strictly positive values on the closed unit interval. In particular

$$
(a+1) /(d-1)=\varphi(1)<1 \text { hence } d-a>2 .
$$

Because of this last inequality the auxiliary mapping

$$
\nu(z):=\frac{\delta z+1}{d} \quad \text { where } \quad \delta:=\sqrt{a d+1}
$$

maps $\mathbb{U}$ into itself; indeed for $z \in \mathbb{U}$ :

$$
|\nu(z)|<\nu(1)=\frac{\delta+1}{d}<1 .
$$

Let $M_{z}$ denote the operator of "multiplication by $z$ " on $H^{2}$ (i.e., the "forward shift"), set $T=C_{\nu} M_{z}^{*}$, and let $P_{0}$ denote the orthogonal projection of $H^{2}$ onto the closed subspace of constant functions:

$$
P_{0} f=\langle f, 1\rangle=f(0) \quad\left(f \in H^{2}\right) .
$$

With this notation we can state what is really the main result of this section:

$$
C_{\varphi}+C_{\varphi}^{*}=P_{0}+C_{\nu}^{*} C_{\nu}+\frac{a}{d} T^{*} T
$$

This decomposition, whose existence was suggested by matrix arguments (see the discussion following the proof of Theorem 6.2 below), exhibits $C_{\varphi}+C_{\varphi}^{*}=2 \operatorname{Re} C_{\varphi}$ as the sum of three positive operators, hence $\operatorname{Re} C_{\varphi}$ is positive. To see that it is strictly positive we need only observe that $C_{\nu}$ is one-to-one (as is any composition operator induced by a non-constant map), hence if $f \in H^{2}$ is not the zero-function then

$$
\left\langle C_{\nu}^{*} C_{\nu} f, f\right\rangle=\left\langle C_{\nu} f, C_{\nu} f\right\rangle=\left\|C_{\nu} f\right\|^{2}>0
$$

i.e., $C_{\nu}^{*} C_{\nu}$ is strictly positive, and therefore the same is true of $\operatorname{Re} C_{\varphi}$.

It remains to prove (20). The idea will be to check that both sides of this equation yield the same result when applied to the reproducing kernels $K_{\lambda}(\lambda \in \mathbb{U})$ introduced in Section 2.2. Because of the reproducing property (2), any function in $H^{2}$ orthogonal to $K_{\lambda}$ vanishes at $\lambda$, hence if $\lambda$ runs through a subset of $\mathbb{U}$ with a limit point in $\mathbb{U}$ then the linear span of the corresponding $K_{\lambda}$ 's is dense in $H^{2}$. Thus it is enough to check our equation on any such 
family of reproducing kernels; for convenience we choose to restrict $\lambda$ to the real interval $(-1,1)$.

First SubClaim: $C_{\nu}^{*} C_{\nu} K_{\lambda}=\frac{d}{d-\lambda} K_{\varphi(\lambda)} \quad(-1<\lambda<1)$.

Proof. A straightforward calculation shows that for $z \in \mathbb{U}$ :

$$
C_{\nu} K_{\lambda}(z)=\frac{d}{d-\lambda} \frac{1}{1-\beta z} \quad \text { where } \quad \beta=\frac{\lambda \delta}{d-\lambda} .
$$

Clearly $\beta>0$, and since $C_{\nu} K_{\lambda} \in H^{2}$ we must also have $\beta<1$, hence the last equation can be rephrased:

$$
C_{\nu}^{*} C_{\nu} K_{\lambda}=\frac{d}{d-\lambda} K_{\nu(\beta)}
$$

Another straightforward calculation shows that

$$
\nu(\beta)=\frac{\lambda a+1}{d-\lambda}=\varphi(\lambda),
$$

which completes the proof of this Subclaim.

SECOnd SubClaim: $T^{*} T K_{\lambda}(z)=\frac{\lambda d z}{d-\lambda} K_{\varphi(\lambda)}$ for each $z \in \mathbb{U}$.

Proof. Once checks easily that $M_{z}^{*} K_{\lambda}=\bar{\lambda} K_{\lambda}$ for each $z \in \mathbb{U}$, so if $1-<\lambda<1$ then $T K_{\lambda}=\lambda C_{\nu} K_{\lambda}$. Thus for $z \in \mathbb{U}$ :

$$
T^{*} T K_{\lambda}(z)=M_{z} C_{\nu}^{*}\left(\lambda C_{\nu} K_{\lambda}\right)(z)=z \lambda C_{\nu}^{*} C_{\nu} K_{\lambda}(z),
$$

which, along with the First Subclaim, yields the desired result.

To complete the proof of Theorem 5.1, fix $\lambda \in(-1,1)$, and note that by the reproducing property (2),

$$
\left(C_{\varphi}+C_{\varphi}^{*}\right) K_{\lambda}=K_{\lambda} \circ \varphi+K_{\varphi(\lambda)}
$$

which, after some routine calculation, results for each $z \in \mathbb{U}$ in:

$$
\begin{aligned}
\left(C_{\varphi}+C_{\varphi}^{*}\right) K_{\lambda}(z) & =1+\frac{d+\lambda a z}{(d-\lambda)-(\lambda a+1) z} \\
& =1+\frac{d+\lambda a z}{d-\lambda} \frac{1}{1-\frac{a \lambda+1}{d-\lambda} z} \\
& =1+\left[\frac{d}{d-\lambda}+\frac{\lambda a z}{d-\lambda}\right] K_{\varphi(\lambda)}(z) \\
& =P_{0} K_{\lambda}(z)+C_{\nu}^{*} C_{\nu} K_{\lambda}(z)+\frac{a}{d} T^{*} T K_{\lambda}(z),
\end{aligned}
$$

where the last line follows from the two Subclaims above. 
5.2. Remarks. (a) In the argument above we borrowed the idea of using reproducing kernels as test functions from Cowen's derivation of the formula for the adjoint of a composition operator induced on $H^{2}$ by a linear fractional transformation [7].

(b) In the final section of this paper we use the argument above to prove strict positivity for $\operatorname{Re} C_{\varphi}$ for a class of composition operators induced by linear fractional maps that fix no point of $\mathbb{U}$.

Theorem 5.1 completes our solution to the Zero-Inclusion Question for composition operators induced by self-maps of $\mathbb{U}$ that fix a point in $\mathbb{U}$. We summarize our work in the following.

5.3. Theorem. Suppose that $\varphi$ is a holomorphic self-map of $\mathbb{U}$ fixing a point $p \in \mathbb{U}$. Then $0 \notin W\left(C_{\varphi}\right)$ if and only if there is an $r \in(0,1]$ such that

$$
|p| \leq \sqrt{r} \text { and } \varphi(z)=\alpha_{p}\left(r \alpha_{p}(z)\right) .
$$

Moreover, $0 \in W\left(C_{\varphi}\right)$ implies $0 \in \operatorname{int} W\left(C_{\varphi}\right)$ as long as $\varphi$ does not have the form $\varphi(z)=t z$ for some $t \in[-1,0]$.

As we have mentioned, if $C_{\varphi}$ is compact, then $\varphi$ fixes a point in $\mathbb{U}$. Because the numerical range of a compact operator is closed if and only if it contains zero, the preceding theorem also provides a characterization of when the numerical range of a compact composition operator is closed. (The compact composition operators on $H^{2}$ are characterized in [31], see also [32, Chapter 10].)

\section{Sectorial Composition Operators}

We call a Hilbert space operator $T$ sectorial provided its numerical range is contained in a sector of the form $\{z:|\arg (z)|<\theta\}$ for some positive acute angle $\theta$ (cf. [14, page 37]). The work of the preceding sections shows that if $\varphi$, not the identity, fixes a point $p \in \mathbb{U}$ then $C_{\varphi}$ can be sectorial only if there is an $r \in(0,1)$ such that $\varphi=\alpha_{p} \circ\left(r \alpha_{p}\right)$ where $|p| \leq \sqrt{r}$. In this section, we prove that, for such a map $\varphi, C_{\varphi}$ is sectorial when $|p|<\sqrt{r}$, but not sectorial when $|p|=\sqrt{r}$.

We begin our work with the case $|p|<\sqrt{r}$. Until further notice, we assume that $r \in(0,1)$ and

$$
\varphi(z)=\alpha_{p}\left(r \alpha_{p}(z)\right)
$$

where $0<p<\sqrt{r}$ (recall there is no loss of generality in assuming $p>0$ ). Hence, as we saw in Section 5,

$$
\varphi(z)=\frac{a z+1}{d-z}
$$

where

$$
a=\frac{r-p^{2}}{p(1-r)} \quad \text { and } \quad d=\frac{1-p^{2} r}{p(1-r)} .
$$

Note well that our assumption $p<\sqrt{r}$ implies $a>0$ and $d>2$. Also note $a<d$. Finally, recall that the auxiliary mapping

$$
\nu(z):=\frac{\delta z+1}{d} \quad \text { where } \quad \delta:=\sqrt{a d+1},
$$


is a self-map of $\mathbb{U}$. Our proof that $C_{\varphi}$ is sectorial depends on representation (20) for $C_{\varphi}+C_{\varphi}^{*}$ and a corresponding representation for $C_{\varphi}-C_{\varphi}^{*}$.

6.1. Lemma. Suppose that $\varphi$ is given by (16) and (17) and that $p<\sqrt{r}$. Then

$$
C_{\varphi}-C_{\varphi}^{*}=\frac{1}{d}\left(C_{\nu}^{*} T-T^{*} C_{\nu}\right)
$$

where $T=C_{\nu} M_{z}^{*}$ and $\nu$ is defined by (19).

Proof. The Lemma may be verified - as was the decomposition (20) - by using the $H^{2}$ reproducing kernels as test functions. What follows is a more informative proof. Let $\psi(z)=\delta z /(d-z)$. Using [7, Theorem 2] we have

$$
C_{\nu}^{*}=M_{d /(d-z)} C_{\psi},
$$

where $M_{d /(d-z)}$ is the operator of multiplication by $d /(d-z)$ on $H^{2}$. The preceding formula and the easily verified relationship $C_{\psi} C_{\nu}=C_{\varphi}$ yield

$$
C_{\varphi}=M_{(d-z) / d} C_{\nu}^{*} C_{\nu}
$$

so that $C_{\varphi}=C_{\nu}^{*} C_{\nu}-(1 / d) T^{*} C_{\nu}$. Hence

$$
C_{\varphi}-C_{\varphi}^{*}=\frac{1}{d}\left(C_{\nu}^{*} T-T^{*} C_{\nu}\right),
$$

as desired.

6.2. Theorem. Suppose that $\varphi$ is given by (16) and (17) and that $p<\sqrt{r}$. Then the numerical range of $C_{\varphi}$ lies in the sector $\{z:|\arg z| \leq \pi / 2-\arctan (a / 2)\}$.

Proof. Let $f$ be an arbitrary unit vector in $H^{2}$ and let $\theta>0$ be such that $\tan (\theta) \leq a / 2$. Observe

$$
2 \operatorname{Re}\left(e^{i \theta}\left\langle C_{\varphi} f, f\right\rangle\right)=\cos (\theta)\left\langle\left(C_{\varphi}+C_{\varphi}^{*}\right) f, f\right\rangle+i \sin (\theta)\left\langle\left(C_{\varphi}-C_{\varphi}^{*}\right) f, f\right\rangle .
$$

Applying the decomposition (20) of $C_{\varphi}+C_{\varphi}^{*}$, we see that the first summand on the right of the preceding equation is greater than or equal to

$$
\cos (\theta)\left(\left\|C_{\nu} f\right\|^{2}+(a / d)\|T f\|^{2}\right) .
$$

Using Lemma 6.1, we obtain the following estimate on the size of the second summand on the right of (21)

$$
\left|\sin (\theta)\left\langle\left(C_{\varphi}-C_{\varphi}^{*}\right) f, f\right\rangle\right| \leq \sin (\theta) \frac{1}{d}\left(\left|\left\langle T f, C_{\nu} f\right\rangle\right|+\left|\left\langle C_{\nu} f, T f\right\rangle\right|\right) .
$$

By the Cauchy-Schwarz Inequality,

$$
\left|\sin (\theta)\left\langle\left(C_{\varphi}-C_{\varphi}^{*}\right) f, f\right\rangle\right| \leq \sin (\theta) \frac{2}{d}\|T f\|\left\|C_{\nu} f\right\|
$$


Suppose that $\left\|C_{\nu} f\right\| \geq\|T f\|$. Then starting with (21) and applying estimates (22) and (23), we have

$$
\begin{aligned}
2 \operatorname{Re}\left(e^{i \theta}\left\langle C_{\varphi} f, f\right\rangle\right) & \geq(a / d) \cos (\theta)\|T f\|^{2}+\cos (\theta)\left\|C_{\nu} f\right\|^{2}-(2 / d) \sin (\theta)\left\|C_{\nu} f\right\|^{2} \\
& =(a / d) \cos (\theta)\|T f\|^{2}+\left\|C_{\nu} f\right\|^{2} \cos (\theta)[1-(2 / d) \tan (\theta)] \\
& \geq(a / d) \cos (\theta)\|T f\|^{2}+\left\|C_{\nu} f\right\|^{2} \cos (\theta)[1-(2 / a) \tan (\theta)] \\
& \geq 0,
\end{aligned}
$$

where we have used $a<d$ to obtain the next-to-last inequality.

Now suppose that $\|T f\| \geq\left\|C_{\nu} f\right\|$. Again, starting with (21) and applying estimates (22) and (23), we have

$$
\begin{aligned}
2 \operatorname{Re}\left(e^{i \theta}\left\langle C_{\varphi} f, f\right\rangle\right) & \geq \cos (\theta)\left\|C_{\nu} f\right\|^{2}+(a / d) \cos (\theta)\|T f\|^{2}-(2 / d) \sin (\theta)\|T f\|^{2} \\
& =\cos (\theta)\left\|C_{\nu} f\right\|^{2}+(a / d)\|T f\|^{2} \cos (\theta)[1-(2 / a) \tan (\theta)] \\
& \geq 0 .
\end{aligned}
$$

We have proved that if we rotate $W\left(C_{\varphi}\right)$ by an angle $\theta$ satisfying $0<\theta \leq \arctan (a / 2)$, the resulting region is still in the closed right half-plane. Since $W\left(C_{\varphi}\right)$ is symmetric with respect to the real axis (see Section 2.7), it follows that $W\left(C_{\varphi}\right)$ lies in the sector

$$
\{z:|\arg z| \leq \pi / 2-\arctan (a / 2)\}
$$

as desired.

Our goal now becomes that of proving $W\left(C_{\varphi}\right)$ is not sectorial when $\varphi$ has the form $\alpha_{p} \circ\left(r \alpha_{p}\right)$ and $p=\sqrt{r}$. We employ matrix methods that contributed to our development of Theorem 5.1. The major element in our proof of Theorem 5.1, the decomposition (20) of $C_{\varphi}+C_{\varphi}^{*}$, evolved from a matrix analysis of the transitional case $p=\sqrt{r}$. For this case $a=0$ in (17) so the expression (16) for $\varphi$ becomes

$$
\varphi(z)=\frac{1}{d-z} \quad \text { with } \quad d=\frac{1+r}{\sqrt{r}}>2,
$$

and the decomposition (20) simplifies to

$$
C_{\varphi}+C_{\varphi}^{*}=P_{0}+C_{\nu} C_{\nu}^{*} \quad \text { where } \quad \nu(z)=\frac{1+z}{d} .
$$

The discussion below is also valid when $d=2$.

For an operator $T$ on $H^{2}$, let us denote its matrix with respect to the orthonormal basis $\left\{z^{j}\right\}_{0}^{\infty}$ by $[T]$. Note that for $\varphi$ as above, $\left[C_{\varphi}\right]$ has as its $j$-th column the MacLaurin coefficient sequence of $\varphi^{j}$. Let $w=1 / d$ and apply the binomial theorem to obtain that the 
$(k, j)$ element of this matrix is:

$$
a_{k, j}:=\left\{\begin{array}{ccc}
\left(\begin{array}{c}
k+j-1 \\
k
\end{array}\right) w^{j+k} & \text { if } & j \geq 1, \\
1 & \text { if } \quad(k, j)=(0,0), \text { and } \\
0 & \text { if } \quad j=0 \text { and } k>0 .
\end{array}\right.
$$

Thus for $j>0$ the $(k, j)$ entry of $\left[C_{\varphi}+C_{\varphi}^{*}\right]$ is

$$
\begin{aligned}
a_{k, j}+a_{j, k} & =\left[\left(\begin{array}{c}
k+j-1 \\
k
\end{array}\right)+\left(\begin{array}{c}
j+k-1 \\
j
\end{array}\right)\right] w^{k+j} \\
& =\left(\begin{array}{c}
k+j \\
k
\end{array}\right) w^{k+j}=\left(\begin{array}{c}
j+k \\
j
\end{array}\right) w^{k+j},
\end{aligned}
$$

a formula which also gives the correct answer for $j=0, k \neq 0$. The only anomaly is the $(0,0)$ entry, which is 2 , instead of the value 1 predicted by the formula. From this we obtain the matrix equation

$$
\left[C_{\varphi}+C_{\varphi}^{*}\right]=\left[P_{0}\right]+T(w)
$$

where $P_{0}$ is, as before, the orthogonal projection of $H^{2}$ onto the space of constant functions $\left(\left[P_{0}\right]\right.$ has $(0,0)$ entry 1 and all other entries zero), and $T(w)$ is the infinite matrix whose $m$-th cross-diagonal is $w^{m}$ times the $m$-th row of Pascal's Triangle $(m=0,1,2, \ldots)$.

The matrix $T(1)$ (which does not correspond to any composition operator) is called Tartaglia's table or Pascal's matrix, and figures in a surprising variety of applications (see [2] for example).

The operator decompositions (25) and (27) yield the matrix factorization (at least for $w \leq 1 / 2)$

$$
T(\alpha)=\left[C_{\nu}\right]^{*}\left[C_{\nu}\right]
$$

with the superscript "**" now denoting matrix transpose. Now $\left[C_{\nu}\right]$, because of the simple form of $\nu$ given in (25), is upper triangular with $w^{j}$ times the $j$-th row of Pascal's triangle as its $j$-th column. Thus (28) furnishes an $L U$-decomposition of $T(w)$.

The analogous decomposition of the $n \times n$ upper left-hand submatrix of $T(w)$ is known (see [5, Theorem 3]), and in fact follows quickly (for $w \leq 1 / 2$ ) from the discussion above and the fact that $C_{\nu}$ leaves invariant the subspace spanned by the monomials $\left\{z^{j}: 0 \leq j \leq n\right\}$. It was, in fact, this result that first led us to the auxiliary mapping $\nu$ and the decomposition (25).

These matrix methods permit us to complete our characterization of sectorial composition operators. For the remainder of the section, we assume

$$
\varphi(z)=\frac{1}{d-z}
$$

where $d \geq 2$. 


\subsection{Theorem. The operator $C_{\varphi}$ is not sectorial.}

We will show that for each positive integer $n$, there is a point in $W\left(C_{\varphi}\right)$ whose argument is $\pi / 2-\pi /(n+1)$. Our proof will ultimately depend on a functional equation satisfied by Chebyshev polynomials of the second kind.

Let $n$ be an arbitrary positive integer, let $t \in \mathbf{R}$, let $M(t)$ be the matrix of $\left(e^{i t} C_{\varphi}+\right.$ $\left.e^{-i t} C_{\varphi}^{*}\right): H^{2} \rightarrow H^{2}$ with respect to the basis $\left\{1, z, z^{2}, \ldots\right\}$ of $H^{2}$, and let $A_{n}(t)$ be the $n \times n$ principal submatrix of $M(t)$. For example,

$$
A_{3}(t)=\left[\begin{array}{ccc}
2 \cos (t) & w \cos (t)+i w \sin (t) & w^{2} \cos (t)+i w^{2} \sin (t) \\
w \cos (t)-i w \sin (t) & 2 w^{2} \cos (t) & 3 w^{3} \cos (t)+i w^{3} \sin (t) \\
w^{2} \cos (t)-i w^{2} \sin (t) & 3 w^{3} \cos (t)-i w^{3} \sin (t) & 6 w^{4} \cos (t)
\end{array}\right],
$$

where $w=1 / d$.

To prove Theorem 6.3 , we show that when $t=\pi /(n+1)$, then $\operatorname{det} A_{n}(t)=0$. Suppose $\operatorname{det} A_{n}(t)=0$; then the numerical range $W\left(A_{n}(t)\right)$ contains 0 , which means 0 also lies in $W\left(e^{i t} C_{\varphi}+e^{-i t} C_{\varphi}^{*}\right)$. In fact, there is a polynomial $p$ of degree $n$ and $H^{2}$ norm 1 such that

$$
\left\langle e^{i t}\left(C_{\varphi}+e^{-i t} C_{\varphi}^{*}\right) p, p\right\rangle=0
$$

(just choose the coefficients of $p$ to form a unit vector in the kernel of $A_{n}(t)$ ). Hence $\operatorname{Re}\left(e^{i t}\left\langle C_{\varphi} p, p\right\rangle\right)=0$, which implies that $\left\langle C_{\varphi} p, p\right\rangle$ lies on the ray $\{z: \arg z=\pi / 2-t\}$ since $\operatorname{Re}\left\langle C_{\varphi} p, p\right\rangle>0$ by Theorem 5.1. Thus, our goal is to prove $\operatorname{det} A_{n}(t)$ vanishes for $t=\pi /(n+1)$.

Let us describe the entries in $A_{n}(t)$, considering the top row of $A_{n}(t)$ and the left-most column of $A_{n}(t)$ to be its 0 -th row and 0 -th column respectively. Using (26), we see that for $0 \leq k \leq n-1$ and $0<j \leq n-1$,

$$
\left[A_{n}(t)\right]_{k, j}=\cos (t) w^{k+j}\left(\begin{array}{c}
k+j \\
j
\end{array}\right)+i \sin (t) w^{k+j} \frac{j-k}{j}\left(\begin{array}{c}
k+j-1 \\
k
\end{array}\right) .
$$

Also,

$$
\left[A_{n}(t)\right]_{0,0}=2 \cos (t), \text { and }\left[A_{n}(t)\right]_{k, 0}=w^{k} \cos (t)-i w^{k} \sin (t) \text { for } k \geq 1 .
$$

Note that for $1 \leq k, j \leq n-1$, each entry in row $k$ of $A_{n}(t)$ contains a factor of $w^{k}$ while each entry in column $j$ contains a factor of the form $w^{j}$. It follows that

$$
\operatorname{det} A_{n}(t)=w^{n(n-1)} \operatorname{det} B_{n}(t)
$$

where $B_{n}(t)$ is free of factors of $w$ :

$$
\left[B_{n}(t)\right]_{k, j}=\cos (t)\left(\begin{array}{c}
k+j \\
j
\end{array}\right)+i \sin (t) \frac{j-k}{j}\left(\begin{array}{c}
k+j-1 \\
k
\end{array}\right)
$$

for $0 \leq k \leq n-1$ and $0<j \leq n-1$. Also the 0 -th column of $B_{n}(t)$ consists of $2 \cos (t)$ followed by $n-1$ entries, each of which is $\cos (t)-i \sin (t)$.

Our goal now becomes that of proving $\operatorname{det} B_{n}(t)=0$ when $t=\pi /(n+1)$. 
6.4. Lemma. For $n \geq 1$,

$$
\operatorname{det} B_{n}(t)=U_{n}(\cos (t))
$$

where $U_{n}$ is the $n$-th Chebyshev polynomial of the second kind.

The polynomial $U_{n}$ is given by

$$
U_{n}(x)=\sum_{k=0}^{[n / 2]}(-1)^{k}\left(\begin{array}{c}
n-k \\
k
\end{array}\right)(2 x)^{n-2 k}
$$

and is defined recursively by

$$
U_{0}=1, U_{1}(x)=2 x \text {, and } U_{n}(x)=2 x U_{n-1}(x)-U_{n-2}(x) \text { for } n \geq 2 .
$$

Moreover, we have the following functional equation

$$
U_{n}(\cos (t))=\frac{\sin ((n+1) t)}{\sin (t)},
$$

[29, p. 7, pp. 39-40]. The preceding equation shows that $U_{n}$ vanishes at $\cos (t)$ whenever $\sin ((n+1) t)=0$ while $\sin (t) \neq 0$. Thus, in particular, $\cos (\pi /(n+1))$ is always a zero of $U_{n}$ (for $\left.n \geq 1\right)$. By the Lemma above $\operatorname{det} B_{n}(\pi /(n+1))=0$. Thus, given the discussion in the paragraphs preceding the statement of Lemma 6.4, we see that Theorem 6.3 follows from the Lemma.

Because we have been unable to find Lemma 6.4 in the literature, we present a detailed proof.

Proof of Lemma 6.4. Note $\operatorname{det} B_{1}(t)=2 \cos (t)=U(\cos (t))$ and $\operatorname{det} B_{2}(t)=4 \cos ^{2}(t)-1=$ $U_{2}(\cos (t))$. Thus the Lemma holds when $n=1$ and $n=2$. To see that it holds for all $n$ we show

$$
\operatorname{det} B_{n}(t)=2 \cos (t) \operatorname{det} B_{n-1}(t)-\operatorname{det} B_{n-2}(t) \text { for } n \geq 3 .
$$

We describe a sequence of elementary row and column operations on $B_{n}(t)$ that will produce a matrix $L_{n}(t)$ whose determinant (which is the same as that of $B_{n}(t)$ ) is easily seen to equal the sum on the right of (30). This will complete the proof of the lemma. The following observations will be used to produce the matrix $L_{n}(t)$.

Observation 1. For $1 \leq k \leq n-2$,

$$
\text { row }(k+1) \text { of } B_{n}(t)-\left(\text { row } k \text { of } B_{n}(t)\right)
$$

equals the row matrix whose first entry is 0 and whose remaining entries constitute the 0 -th through $(n-2)$-nd entries of row $(k+1)$ of $B_{n}(t)$.

In other words, if row $k$ of $B_{n}(t)$ is subtracted from row $k+1$, the result is the "forward shift" of row $(k+1)$ with the last entry dropped. In symbols this difference equals

$$
\left[0 R_{k+1}\right] \text {, }
$$

where $R_{k+1}$ represents row $(k+1)$ of $B_{n}(t)$ with its final entry truncated.

Observation 2. For $1 \leq k, j \leq n-1$,

$$
(-1)\left[B_{n}(t)\right]_{k, j-1}+\left[B_{n}(t)\right]_{k, j}=\left[B_{n}(t)\right]_{k-1, j}
$$


Apply Observation 1 repeatedly, first replace row $(n-1)$ of $B_{n}(t)$ with the difference

$$
\text { row }(n-1) \text { of } B_{n}(t)-\left(\text { row }(n-2) \text { of } B_{n}(t)\right) \text {; }
$$

then replace row $(n-2)$ with the difference of rows $(n-2)$ and $(n-3)$, and continue this process until row 2 has been replaced with the difference row 2 - row 1 . (Recall that the top row of $B_{n}(t)$ is row 0 .) What results is the matrix

$$
\left[\begin{array}{ccccc}
2 \cos (t) & \cos (t)+i \sin (t) & \cos (t)+i \sin (t) & \cdots & \cos (t)+i \sin (t) \\
\cos (t)-i \sin (t) & 2 \cos (t) & 3 \cos (t)+i \sin (t) & \cdots & n \cos (t)+i(n-2) \sin (t) \\
0 & R_{2} & & & \\
0 & R_{3} & & & \\
\vdots & \vdots & \vdots & \vdots & \vdots \\
0 & R_{n-1} & & &
\end{array}\right]
$$

which we label $E_{n}(t)$. Now apply Observation 2 repeatedly to $E_{n}(t)$, first replace its $(n-1)$ column of with the difference

$$
\text { column }(n-1) \text { of } E_{n}(t)-\left(\operatorname{column}(n-2) \text { of } E_{n}(t)\right) \text {; }
$$

then replace column $(n-2)$ with the difference of columns $(n-2)$ and $(n-3)$, and continue this process until column 2 has been replaced with the difference column $2-$ column 1 . What results is the matrix

$$
\left[\begin{array}{ccccc}
2 \cos (t) & \cos (t)+i \sin (t) & 0 & \cdots & 0 \\
\cos (t)-i \sin (t) & 2 \cos (t) & \cos (t)+i \sin (t) & \cdots & \cos (t)+i \sin (t) \\
0 & R_{1} & & & \\
0 & R_{2} & & & \\
\vdots & \vdots & \vdots & \vdots & \vdots \\
0 & R_{n-2} & & &
\end{array}\right],
$$

which we label $L_{n}(t)$. Calculate $\operatorname{det} L_{n}(t)$ by expanding along the first column of $L_{n}(t)$ :

$$
\operatorname{det} L_{n}(t)=2 \cos (t) \operatorname{det} B_{n-1}(t)-e^{-i t} \operatorname{det}\left[\begin{array}{cccc}
\cos (t)+i \sin (t) & 0 & \cdots & 0 \\
R_{1} & & & \\
R_{2} & & & \\
\vdots & \vdots & \vdots & \vdots \\
R_{n-2} & & &
\end{array}\right] \text {. }
$$

Now apply Observations 1 and 2, exactly as they were applied to $B_{n}(t)$, to reduce the matrix on the right of the preceding display to

$$
\left[\begin{array}{cccc}
\cos (t)+i \sin (t) & 0 & \cdots & 0 \\
0 & \widetilde{R}_{0} & & \\
0 & \widetilde{R}_{1} & & \\
\vdots & \vdots & \vdots & \vdots \\
0 & \widetilde{R}_{n-3} & &
\end{array}\right]
$$


where for $k=0,1, \ldots, n-3, \widetilde{R}_{k}$ is the $k$-th row of $B_{n}(t)$ with its final two entries truncated. This reduced matrix permits us to complete our calculation (31):

$$
\begin{aligned}
\operatorname{det} L_{n}(t) & =2 \cos (t) \operatorname{det} B_{n-1}(t)-e^{-i t} e^{i t} \operatorname{det}\left[\begin{array}{c}
\widetilde{R}_{0} \\
\widetilde{R}_{1} \\
\vdots \\
\widetilde{R}_{n-3}
\end{array}\right] \\
& =2 \cos (t) \operatorname{det} B_{n-1}(t)-\operatorname{det} B_{n-2}(t),
\end{aligned}
$$

as desired.

We have proved Theorem 6.3 by showing that when $\varphi(z)=1 /(d-z)$ for $d \geq 2, W\left(C_{\varphi}\right)$ contains points in the first quadrant arbitrary close to the imaginary axis. Recall from Section 2.7, that since $\varphi$ is real on the real axis, $W\left(C_{\varphi}\right)$ is symmetric with respect to the real axis; hence, Theorem 6.3 shows that 0 is not a corner point of $\bar{W}\left(C_{\varphi}\right)$. We discuss corner points in detail in the next section.

\section{Corner Points}

An element $p$ of a set $E \subset \mathbb{C}$ is a corner point of $E$ provided $E$ is contained in a half-cone with vertex $p$ and angular opening less than $\pi$. It is well known that for any Hilbert space operator $T$, a corner point of $W(T)$ must be an eigenvalue of $T([10])$. A corner point of $\bar{W}(T)$ must be an approximate eigenvalue; in particular, any corner point of $\bar{W}(T)$ must belong to the spectrum of $T([20])$.

Corner points of numerical ranges of composition operators are discussed in [26], which contains, e.g., the following result: if $C_{\varphi}$ is compact with $\varphi$ a nonconstant function satisfying $\varphi(0)=0$ and $\varphi^{\prime}(0)=0$, then 0 is an interior point of $W\left(C_{\varphi}\right)$ and 1 is the only possible corner point of $W\left(C_{\varphi}\right)$ (which equals $\bar{W}\left(C_{\varphi}\right)$ because $0 \in W\left(C_{\varphi}\right)$ and $C_{\varphi}$ is compact). Here, we obtain a complete characterization of corner points $\bar{W}\left(C_{\varphi}\right)$ when $C_{\varphi}$ is compact.

We begin with the simpler task of characterizing the corner points not of $\bar{W}\left(C_{\varphi}\right)$ but of $W\left(C_{\varphi}\right)$, under the assumption that $\varphi$ fix a point $p$ in $\mathbb{U}$. These corner points must be in the point spectrum of $C_{\varphi}$, which is known to be a subset of $\left\{\varphi^{\prime}(p)^{n}: n=0,1,2, \ldots\right\}$ ([6, Theorem 4.1]).

In the proof of [26, Theorem 3.6], Matache shows that if $\varphi$ induces a compact operator and fixes a point $p \in \mathbb{U} \backslash\{0\}$ such that $p$ doesn't belong to the iterate sequence $\left(\varphi^{[n]}(0)\right)$, then the set $\left\{\varphi^{\prime}(p)^{n}: n \geq 1\right\}$ belongs to the interior of $W\left(C_{\varphi}\right)$. The following Theorem shows that the restrictions of compactness and $p \notin\left\{\varphi^{[n]}(0): n \geq 1\right\}$ may be removed. We do not consider composition operators induced by dilations and rotations since the numerical ranges for such operators are easily described (Sections 2.8 and 2.9).

7.1. Theorem. Suppose $\varphi$ is a self-map of $\mathbb{U}$ that is neither a dilation nor a rotation and $\varphi(p)=p$ for some $p \in \mathbb{U}$. Then $\left\{\varphi^{\prime}(p)^{n}: n \geq 1\right\}$ is contained in the interior of $W\left(C_{\varphi}\right)$.

Proof. If $\varphi^{\prime}(p)=0$, the Theorem follows immediately from Corollary 3.6 because $\varphi$ is not univalent. Thus we assume $\varphi^{\prime}(p) \neq 0$. 
In case $p=0$, the proof of Theorem 3.8 shows that there is a nonzero number $b$ and a positive integer $m$ such the compression of $C_{\varphi}$ to the two-dimensional subspace of $H^{2}$ with basis $\left\{z^{n}, z^{n+m}\right\}$ has matrix

$$
\left[\begin{array}{cc}
\varphi^{\prime}(0)^{n} & 0 \\
n b \varphi^{\prime}(0)^{n} & \varphi^{\prime}(0)^{n+m}
\end{array}\right]
$$

Because the entry in the first column and second row is always nonzero, the numerical range of $C_{\varphi}$ contains a nondegenerate ellipse with foci $\lambda^{n}$ and $\lambda^{n+m}$ for each $n \geq 1$. Thus $\left\{\varphi^{\prime}(0)^{n}: n \geq 1\right\} \subset$ int $W\left(C_{\varphi}\right)$, as desired.

In case $p \neq 0$, the work of Section 4 shows that for each $n \geq 0$, there is an an orthonormal set $\left\{b_{n}, b_{n+1}\right\}$ in $H^{2}$ generating a subspace $\mathcal{M}$ of $H^{2}$ such that the compression of $C_{\varphi}$ to $\mathcal{M}$ has matrix

$$
\left[\begin{array}{cc}
\varphi^{\prime}(p)^{n} & 0 \\
\left\langle C_{\varphi} b_{n}, b_{n+1}\right\rangle & \varphi^{\prime}(p)^{n+1}
\end{array}\right]
$$

By Corollary 4.2 , the $(2,1)$ entry of this matrix can be 0 for at most one positive integer $n_{0}$ (we have already observed, in the proof of Proposition 4.3 that it is not zero if $n=0$ ). Thus, for all nonnegative integers $n \neq n_{0}$, we see that $W\left(C_{\varphi}\right)$ contains a nondegenerate ellipse with foci $\varphi^{\prime}(p)^{n}$ and $\varphi^{\prime}(p)^{n+1}$. Hence for each positive integer $n$, including $n_{0}, \varphi^{\prime}(p)^{n}$ is a focal point of a nondegenerate ellipse contained in $W\left(C_{\varphi}\right)$, so that the entire sequence $\left\{\varphi^{\prime}(p)^{n}: n \geq 1\right\}$ lies in the interior of $W\left(C_{\varphi}\right)$, as desired.

7.2. Corollary. Suppose $\varphi$ is a self-map of $\mathbb{U}$ that is neither a dilation nor a rotation and $\varphi(p)=p$ for some $p \in \mathbb{U}$. Then 1 is the only possible corner point of $W\left(C_{\varphi}\right)$.

Proof. All eigenvalues of $C_{\varphi}$ except for 1 lie in the interior of $W\left(C_{\varphi}\right)$ by Theorem 7.1.

Observe that 1 is a corner point of $W\left(C_{\varphi}\right)$ if and only if it is a corner point of $\bar{W}\left(C_{\varphi}\right)$. Thus we complete our characterization of the corner points of $W\left(C_{\varphi}\right)$ by answering the following.

When is 1 a corner point of $\bar{W}\left(C_{\varphi}\right)$ ?

If $\varphi(0) \neq 0$, then 1 is not a normal eigenvalue of $C_{\varphi}$; hence by Theorem 3.4 , the point 1 belongs to $\in \operatorname{int} W\left(C_{\varphi}\right)$, and therefore cannot be a corner point of $\bar{W}\left(C_{\varphi}\right)$. (This observation is a generalization of Theorem 3.3 in [26].) Thus we assume that $\varphi(0)=0$. Then the subspace $H_{0}^{2}$ is invariant for $C_{\varphi}$ and has as its orthogonal complement the space of constant functions. It is known that $\varphi$ is an inner function (i.e. $\varphi$ has radial limits of modulus 1 a.e. on $\partial \mathbb{U})$ if and only if the restriction of $C_{\varphi}$ to $H_{0}^{2}$ has norm 1 [33, Theorem 5.1]. Thus if $\varphi$ is not inner then $\bar{W}\left(C_{\varphi} \mid H_{0}^{2}\right)$ lies in the closed disc with center at the origin and radius $\left\|\left.C_{\varphi}\right|_{H_{0}^{2}}\right\|<1$, and therefore $\bar{W}\left(C_{\varphi}\right)$ itself lies in the convex hull of this disc and the singleton $\{1\}$. In particular 1 is a corner point of $\bar{W}\left(C_{\varphi}\right)$.

Conversely suppose $\varphi$ is inner, fixes the origin, and is not a rotation. Then $C_{\varphi}$ is a nonunitary isometry taking $H^{2}$ properly into itself, and the spectrum of any such operator is well known to be the closed unit disc $\overline{\mathbb{U}}$. (Proof: Because $T$ is an isometry $\sigma(T) \subset \overline{\mathbb{U}}$. Also, $T^{*} T=I$, so for each $\lambda \in \mathbb{U}$ there is the factorization $T-\lambda I=\left(I-\lambda T^{*}\right) T$ where $\left\|\lambda T^{*}\right\|=|\lambda|\left\|T^{*}\right\|=|\lambda|<1$. Thus $I-\lambda T^{*}$ is invertible, but $T$ is not, so neither is $T-\lambda I$. Thus $\lambda \in \sigma(T)$, which shows that $\mathbb{U} \subset \sigma(T)$.) 
Now $\bar{W}\left(C_{\varphi}\right)$ must contain the spectrum, but must also lie in the $\overline{\mathbb{U}}$, so it coincides with $\overline{\mathbb{U}}$. In particular 1 is not a corner point of $\bar{W}\left(C_{\varphi}\right)$ in this case. To summarize:

7.3. Theorem. If $\varphi(0) \neq 0$, then $1 \in \operatorname{int} W\left(C_{\varphi}\right)$. If $\varphi(0)=0$ and $\varphi$ is not a rotation, then 1 is a corner point of $\bar{W}\left(C_{\varphi}\right)$ if and only if $\varphi$ is not inner.

We are now in a position to characterize the corner points of $\bar{W}\left(C_{\varphi}\right)$ for compact $C_{\varphi}$. Note well that when $C_{\varphi}$ is compact, $\varphi$ cannot be inner: if $\varphi$ is inner, then the $H^{2}$ norm of $\varphi^{n}$ is 1 for each $n \geq 1$ so that $C_{\varphi}$ does not take the sequence $\left(z^{n}\right)$ in $H^{2}$, which converges weakly to 0 , to a sequence that converges in norm to 0 . In particular, the symbol of a compact composition operator cannot be a rotation mapping. Since the spectrum of a compact operator consists of its eigenvalues together with 0 and since only spectral points can be corner points, to obtain the full story on corner points of a compact operator we need only check the origin and the eigenvalues of the operator. Thus for a compact composition operator $C_{\varphi}$, the set of points that must be checked is $\left\{\varphi^{\prime}(p)^{n}: n \geq 0\right\} \cup\{0\}$ where $p \in \mathbb{U}$ is the point fixed by $\varphi$. (Recall that the symbol of compact composition operator must fix a point in $\mathbb{U}$.) We have seen (Theorem 7.1) that when $\varphi$ fixes $p \in \mathbb{U}$ and is neither a dilation nor a rotation, then points $\left\{\varphi^{\prime}(p)^{n}: n \geq 1\right\}$ belong to the interior of $W\left(C_{\varphi}\right)$, which yields:

7.4. Corollary. The numbers 1 and 0 are the only possible corner points of $\bar{W}\left(C_{\varphi}\right)$ when $C_{\varphi}$ is compact and $\varphi$ is not a dilation.

7.5. Theorem. Let $\varphi$ be a self-map of $\mathbb{U}$ that induces a compact composition operator on $H^{2}$.

(a) Suppose that $\varphi(z)=\beta z$, where $|\beta|<1$. If $0 \leq \beta<1$, then 0 and 1 are the corner points of $\bar{W}\left(C_{\varphi}\right)$; otherwise, the set of corner points of $\bar{W}\left(C_{\varphi}\right)$ has the form $\left\{\beta^{n}: n=\right.$ $0,1, \ldots k-1\}$ where $k$ is the least positive integer such that $\beta^{k}$ lies in the convex hull of $\left\{\beta^{n}: n=0,1, \ldots k-1\right\}$.

(b) If $\varphi(0)=0$ and $\varphi$ is not a dilation, then 1 is the only corner point of $\bar{W}\left(C_{\varphi}\right)$.

(c) If $\varphi$ fixes a point in $\mathbb{U} \backslash\{0\}$ and is not a positive conformal dilation, then $\bar{W}\left(C_{\varphi}\right)$ has no corner points.

(d) If $\varphi$ is the positive conformal dilation given by $\varphi(z)=\alpha_{p}\left(r \alpha_{p}(z)\right)$, where $0<r,|p|<1$, then $\bar{W}\left(C_{\varphi}\right)$ has no corner points when $|p| \geq \sqrt{r}$ and has 0 as its only corner point when $|p|<r$.

Proof. The proof of (a) follows immediately from the discussion of Section 2.8.

Suppose the hypothesis of (b) holds. Then Theorem 5.3 shows that 0 belongs to the interior of $W\left(C_{\varphi}\right)$; hence, by Corollary 7.4, 1 is the only possible corner point of $\bar{W}\left(C_{\varphi}\right)$. That 1 is a corner point in this situation follows from Theorem 7.3 because the symbol of a compact composition operator cannot be inner.

Suppose that hypothesis of (c) holds. Then, reasoning just as in the preceding paragraph, we see that 1 is the only possible corner point. However, 1 is not a corner point in this situation: in fact 1 must lie in the interior of $W\left(C_{\varphi}\right)$ because 1 is not an normal eigenvalue $(\varphi(0) \neq 0$ since $\varphi$ fixes a nonzero point in $\mathbb{U})$.

That (d) holds follows quickly from the results of Section 6 and the observation that 1 is, again, not a normal eigenvalue. 
We remark that Corollary 7.4 and Theorem 7.5 are actually valid for Riesz composition operators. A Riesz operator is spectrally indistinguishable from a compact operator. For a discussion of Riesz composition operators, the reader may consult, e.g., [3].

\section{No Fixed Point in $\mathbb{U}$}

We have answered the Zero-Inclusion Question for composition operators whose symbols fix a point in $\mathbb{U}$. What about those whose symbols don't fix a point in $\mathbb{U}$ ? If $\varphi$ fails to fix a point in $\mathbb{U}$, then, by the Denjoy-Wolff Theorem, $\varphi$ has an attractive fixed point $p \in \partial \mathbb{U}$. Moreover, $\varphi^{\prime}(p)=\lim _{r \rightarrow 1^{-}} \varphi^{\prime}(r p)$ exists and $0<\varphi^{\prime}(p) \leq 1$ (see, [32, Chapter 5], e.g.). The mapping $\varphi$ is said to be of hyperbolic type, when $\varphi^{\prime}(p)<1$; if $\varphi^{\prime}(p)=1$ and the iterate sequence $\left(\varphi^{[n]}(0)\right)$ is separated in the hyperbolic metric on $\mathbb{U}$, then $\varphi$ is of parabolic automorphism type; finally, if $\varphi^{\prime}(p)=1$ and the iterate sequence $\left(\varphi^{[n]}(0)\right)$ is not separated in the hyperbolic metric on $\mathbb{U}$, then $\varphi$ is of parabolic nonautomorphism type.

When $\varphi$ is of hyperbolic type or parabolic automorphism type, then the spectrum of the corresponding composition operator must contain at least the unit circle ([6, Theorems 4.5 and Theorem 4.10]), which, by convexity, puts 0 in the interior of $W\left(C_{\varphi}\right)$. However, as we describe below, when $\varphi$ is of parabolic nonautomorphism type, the work of Section 5 shows that zero need not belong to $W\left(C_{\varphi}\right)$.

Write $\sigma(z)=(1+z) /(1-z)$, a conformal mapping taking $\mathbb{U}$ onto the open right halfplane RHP, the unit circle onto the imaginary axis, and sending 1 to $\infty$ and -1 to 0 . Fix $t$, initially in the closed right half-plane, and consider the parabolic map $\Phi(w)=w+t$, which takes RHP into itself. If $t$ is pure imaginary then our translation map is a conformal automorphism of RHP, but otherwise it is not. Thus $\varphi_{t}(z):=\sigma^{-1}(\sigma(z)+t)$ is a parabolic automorphism of $\mathbb{U}$ precisely when $t$ is pure imaginary, and a parabolic non-automorphic self-map of $\mathbb{U}$ when $\operatorname{Re} t>0$.

In case $t$ is not real the spectrum of $C_{\varphi_{t}}$ is known to be an origin-centered disc in the automorphic case [27] and a curve spiraling into the origin in the non-automorphic case [6, Section 6]. In either case, these spectral considerations place the origin in the interior of $W\left(C_{\varphi_{t}}\right)$. However if $t$ is real (so $t>0$ ) then the spectrum of $C_{\varphi_{t}}$ is just the closed unit interval ([6, Section 6] again), so for these operators there is a chance that the origin may not lie in the numerical range. This is exactly what happens!

Upon writing out the formula for $\varphi_{t}$ one obtains (16),

but now with

$$
\varphi(z)=\frac{a z+1}{d-z}
$$

$$
a=\frac{2}{t}-1 \quad \text { and } \quad d=\frac{2}{t}+1 .
$$

For $0<t \leq 2$ both $a$ and $d$ are non-negative, and the map $\nu$ defined by (19) becomes simply

$$
\nu(z)=\frac{2 z+t}{2+t} \quad(z \in \mathbb{U}),
$$

which obviously takes $\mathbb{U}$ into itself. Now the argument that led to the decomposition (20) works once again, with no changes whatsoever, in this new situation, and shows that $\operatorname{Re} C_{\varphi_{t}}$ is strictly positive definite whenever $0<t \leq 2$. For $t>2$ the origin lies in the interior of 
$W\left(C_{\varphi_{t}}\right)$, as an analysis exactly like that of Theorem 5.1 (c) $\rightarrow$ (b) shows (with eigenvectors $e^{-s \sigma}$, where $s$ is positive and large).

Because any linear fractional mapping $\varphi$ of parabolic nonautomorphism type is, by rotational conjugation, equivalent to one of the mappings $\varphi_{t}$ discussed above, we have answered the Zero-Inclusion Question for all composition operators except those with univalent, non linear-fractional symbols that are of parabolic nonautomorphism type.

Suppose $\varphi$ is of parabolic nonautomorphism type and is not linear fractional. Must $0 \in W\left(C_{\varphi}\right)$ ?

\section{REFERENCES}

[1] J. Agler, Geometric and topological properties of the numerical range, Indiana Univ. Math. J. 31 (1982), $767-777$.

[2] M. F. Aburdene, J. E. Dorband, Unification of Legendre, Laguerre, Hermite, and binomial discrete transforms using Pascal's matrix, Multidimens. Systems Signal Process. 5 (1994), 301-305.

[3] P. S. Bourdon and J. H. Shapiro, Riesz composition operators, Pacific J. Math. 181 (1997), 231-246.

[4] P. S. Bourdon and J. H. Shapiro, The numerical ranges of automorphic composition operators, J. Math. Analysis and Appl., to appear.

[5] R. Brawer and M. Pirovino, The linear algebra of the Pascal matrix, Linear Alg. and Appl. 174 (1992), $13-23$

[6] C. C. Cowen, Composition operators on $H^{2}$, Journal of Operator Theory, 9 (1983), 77-105.

[7] C. C. Cowen, Linear fractional composition operators on $H^{2}$, Integral Equations and Operator Theory, 11 (1988), 151-160.

[8] C. C. Cowen and B. D. MacCluer, Composition Operators on Spaces of Analytic Functions, CRC Press, Boca Raton, 1995.

[9] C. Davis, The Toeplitz-Hausdorff theorem explained, Canad. Math. Bull 14 (1971), 245-246.

[10] W. F. Donoghue, On the numerical range of a bounded operator, Mich. Math. J. 4 (1957), 261-263.

[11] G. de Barra, J. R. Giles, and B. Sims, On the numerical range of compact operators on Hilbert spaces, J. London Math. Soc. 5 (1972), 704-706.

[12] N. P. Dekker, Joint Numerical Range and Joint Spectrum of Hilbert Space Operators, Thesis, Free University of Amsterdam, 1969.

[13] P. L. Duren, Theory of $H^{p}$ Spaces, Academic Press, 1970.

[14] J. A. Goldstein, Semigroups of linear operators and applications, Oxford University Press, 1985.

[15] K. E. Gustafson and D. K. M. Rao, Numerical Range, The Field of Values of Linear Operators and Matrices, Springer, New York, 1997.

[16] J. Guyker, On reducing subspaces of composition operators, Acta Sci. Math. (Szeged) 53 (1989), 369376.

[17] F. Jafari et al., editors, Studies on Composition Operators, Contemp. Math. Vol. 213, American Math. Soc. 1998.

[18] P. R. Halmos, A Hilbert Space Problem Book, Second Edition, Springer, New York 1982.

[19] F. Hausdorff, Der Wertvorrat einer Bilinearform, Math. Z. 3 (1919), 314-316.

[20] S. Hildebrandt, Über den Numerischen Wertebereich eines Operators, Math. Ann. 163 (1966), $230-247$.

[21] R. A. Horn and C. R. Johnson, Topics in Matrix Analysis, Cambridge Univ. Press 1991.

[22] C. R. Johnson, Normality and the numerical range, Lin. Alg. App. 15 (1976) 89-94.

[23] G. H. Lawden, Pascal matrices, Math. Gaz. 56 (1972), 325-327.

[24] J. E. Littlewood, On inequalities in the theory of functions, Proc. London Math. Soc. 23 (1925), $481-519$.

[25] F. Malmquist, Sur la détermination d'une classe de fonctions analytiques par leurs valeurs dans un enseble donné de points. Comptes Rendus du Sixième Congrès (1925) des Mathématiciens Scandinaves, Kopenhagen 1926, 253-259.

[26] V. Matache, Numerical ranges of composition operators, preprint 1999.

[27] E. A. Nordgren, Composition operators, Canadian J. Math. 20 (1968), 442-449.

[28] P. Poggi-Corradini, The Hardy class of Koenigs maps, Michigan Math. J. 44 (1997), 495-507. 
[29] T. J. Rivlin, Chebyshev Polynomials, Second Edition, John Wiley \& Sons, 1990

[30] W. Rudin, Real and Complex Analysis, Third edition, McGraw-Hill, New York, 1987.

[31] J. H. Shapiro, The essential norm of a composition operator, Annals of Math. 125 (1987), 375-404.

[32] J. H. Shapiro, Composition Operators and Classical Function Theory, Springer Verlag, 1993.

[33] J. H. Shapiro, What do composition operators know about inner functions?, Monatshefte für Mathematik 130 (2000), 57-70.

[34] O. Toeplitz, Das algebraische Analogon zu einem Satz von Fejér, Math. Z. 2 (1918), 187-197.

[35] J. L. Walsh, Interpolation and Approximation by Rational Functions in the Complex Domain, American Math. Soc., 1935

[36] J. P. Williams, Similarity and the numerical range, J. Math. Anal. App. 26 (1969), 307-314.

WAShington AND LeE University, LeXington, VA 24450, USA

E-mail address: pbourdon@wlu.edu

Michigan State University, East Lansing, MI 48824-1027, USA

E-mail address: shapiro@math.msu.edu 